\title{
A Prediction-Based Approach to Distributed Filtering With Missing Measurements and Communication Delays Through Sensor Networks
}

\author{
Jun $\mathrm{Hu}^{(}{ }^{(}$, Member, IEEE, Zidong Wang ${ }^{(}$, Fellow, IEEE, Guo-Ping Liu ${ }^{(}$, Fellow, IEEE, \\ Hongxu Zhang, and Rukshan Navaratne
}

\begin{abstract}
This article addresses the prediction-based distributed filtering problem for a class of time-varying nonlinear stochastic systems with communication delays and missing measurements through the sensor networks. The phenomenon of the missing measurements is depicted by a set of Bernoulli distributed random variables, where each sensor node possesses its own missing probability. The communication delays are taken into account, which commonly occur during the estimation exchanges among the sensor nodes with communication links. A new prediction-based suboptimal distributed filter is designed by taking the missing probabilities and the prediction estimation into account, which has the advantages on the active compensation of the impacts caused by the missing measurements and communication delays. That is, a new compensation filtering method within the time-varying framework is presented based on the predictive estimation and the innovation measurements. A locally minimum upper bound matrix for the estimation error covariance is obtained by properly designing the distributed filter gain
\end{abstract}

Manuscript received October 24, 2019; accepted December 26, 2019. This work was supported in part by the National Natural Science Foundation of China under Grant 61673141, Grant 61873148, Grant 61933007, and Grant 61773144, in part by the European Regional Development Fund and Sêr Cymru Fellowship under Grant 80761-USW-059, in part by the Outstanding Youth Science Foundation of Heilongjiang Province of China under Grant JC2018001, in part by the Fundamental Research Foundation for Universities of Heilongjiang Province, and in part by the Alexander von Humboldt Foundation of Germany. This article was recommended by Associate Editor M. Basin. (Corresponding author: Jun Hu.)

Jun $\mathrm{Hu}$ is with the School of Engineering, University of South Wales, Pontypridd CF37 1DL, U.K., and also with the Department of Mathematics, Harbin University of Science and Technology, Harbin 150080, China (e-mail: hujun2013@gmail.com).

Zidong Wang is with the Department of Computer Science, Brunel University London, Uxbridge UB8 3PH, U.K. (e-mail: zidong.wang@brunel.ac.uk).

Guo-Ping Liu is with the School of Engineering, University of South Wales, Pontypridd CF37 1DL, U.K., and also with the Department of Artificial Intelligence and Automation, Wuhan University, Wuhan 430072, China (e-mail: guoping.liu@ southwales.ac.uk).

Hongxu Zhang is with the School of Measurement Control Technology Communication Engineering and Heilongjiang Provincial Key Laboratory of Optimization Control and Intelligent Analysis for Complex Systems, Harbin University of Science and Technology, Harbin 150080, China (e-mail: hongxuzhang@hrbust.edu.cn).

Rukshan Navaratne is with the School of Engineering, University of South Wales, Pontypridd CF37 1DL, U.K. (e-mail: rukshan.navaratne@southwales.ac.uk).

Color versions of one or more of the figures in this article are available online at http://ieeexplore.ieee.org.

Digital Object Identifier 10.1109/TSMC.2020.2966977 at every sampling step. Furthermore, the performance analysis problem of the prediction-based distributed filtering algorithm is discussed by providing the desirable theoretical derivations. Finally, some comparative simulations are used to show the advantages of the presented prediction-based distributed filtering strategy under delay compensation mechanism.

Index Terms-Communication delays, missing measurements, monotonicity discussions, nonlinear stochastic systems, prediction-based distributed filtering.

\section{INTRODUCTION}

$\mathbf{S}$ ENSOR networks include a large number of spatially dispersed sensors with certain topological structure, which have broad applications in reality, including the forest fire detection, water quality monitoring, the natural disaster prevention, and so on [11], [12], [43]. For the multisources data collected by the sensors, the key problem is how to design proper filtering schemes based on the local information shared by the adjacent nodes [2], [35]. Over the past decade, a great amount of filtering methods in the centralized or distributed way have been proposed in [37] and [42]. Compared with the centralized filtering strategy, the distributed data processing approaches have provided efficient estimation schemes in the parallel way to improve the filtering performance and the fault tolerance capability [1], [26], [41]. Recently, some state estimation algorithms have been proposed by taking certain evaluation criteria into consideration [14], [15], [24], [39]. For example, the distributed optimal filtering approaches based on the innovation analysis technique have been given in [3] and [4] for networked multisensor systems and a varianceconstrained distributed filtering algorithm has been presented in [7] based on the information affected by the deception attack and uniform quantization. In [9], a novel distributed filtering approach with guaranteed $H_{\infty}$ performance has been given to attenuate the influences from uncertain observations, including the quantized measurements and data packet dropouts. Very recently, both the network security and the robustness of algorithm have been considered in [6], and a new resilient variance-constrained filtering method in a distributed manner has been developed for power systems with cyber-attacks. 
Among the design of control and filtering algorithms in the networked environments, a great deal of effort has been made to examine the impacts from the missing measurements or packet dropouts onto the whole system performance [5], [17], [18], [34], [44]. Accordingly, some efficient filtering methods under different criteria have been reported [16], [32], [38], [46]. For example, the locally optimal estimation method based on the orthogonal projection principle has been presented in [47] for discrete-time linear stochastic systems with missing measurements over wireless sensor networks, and a fusion estimation method has been proposed in terms of certain fusion rule of the matrix weights. In order to enhance the fault-tolerant capability of the estimation techniques, the optimal fusion filtering algorithms in a distributed manner have been proposed in [27] and [28] for linear discrete time-varying stochastic systems with missing measurements, where a distributed fusion estimation scheme has been presented in [28] by employing the matrix-weighted fusion idea to provide the compensation mechanism. Besides, considerable attention has been made to develop the nonlinear distributed filtering methods with the hope to attenuate the degradations induced by the missing measurements [8], [31]. In particular, the distributed filtering approaches under the $H_{\infty}$ performance constraints have been given in [32] and [40] for time-invariant nonlinear stochastic systems subject to missing measurements through the network transmissions. In [8], a robust distributed filtering algorithm with the average $H_{\infty}$ performance requirement has been designed for time-varying nonlinear stochastic systems subject to randomly varying nonlinearities through the lossy sensor networks. However, it is worthwhile to notice that few methods can be applicable for handling the optimized distributed filtering issue under variance constraint of time-varying nonlinear multisensor systems subject to degraded measurements.

On the other hand, it is well recognized that the communication delays should be addressed carefully during the design of a distributed filter or controller, otherwise the estimation accuracy and efficiency would be degraded [20], [25], [29], [30], [36]. Recently, some fusion filtering schemes in the distributed way have been developed for stochastic systems with communication delays as in [27] under the minimum variance constraint and in [33] within the receding horizon framework, where the matrix-weighted fusion criterion has been utilized to fuse the local estimations. In [10], an event-based distributed filtering method with the weighting average $H_{\infty}$ performance requirement has been proposed, where the coordination design problem of filter parameters and triggered thresholds has been carried out. Nevertheless, it should be mentioned that most existing distributed filtering schemes dealing with the communications delays have been proposed in terms of the out-of-date estimation directly, which indeed degrades the filtering efficiency and accuracy. In order to actively compensate the communication delays, some forward predictive mechanisms have been presented in [19], [21], and [22] to handle the control synthesis problems for systems with networked transmissions. In contrast to the predictive control methods, however, few results with the active compensation mechanism are available for tackling the distributed filtering problems subject to communication delays. Very recently, the predictive compensation idea has been initially introduced to handle the estimation/filtering problem with communication delays, where new distributed algorithms have been developed for time-invariant linear systems in [48] and time-varying linear systems in [13], respectively. To the best of our knowledge, the predictionbased distributed filtering problem for time-varying stochastic nonlinear systems subject to degraded measurements has not been fully studied, which needs additional research attentions to provide a new method in order to actively compensate the impacts caused by the communication delays and missing measurements.

In this article, we aim to tackle the locally optimal distributed filtering problem for time-varying nonlinear stochastic systems subject to communication delays and missing measurements over sensor networks. First, the possible communication delays during the estimation exchanges between linked senor nodes are considered and a prediction-based updating rule is developed to actively provide the state estimate at current time step based on the delayed estimation. That is, the prediction-based estimations of adjacent sensor nodes at the updating instant are obtained in terms of the delayed transmissions. Second, the distributed filter of the recursive feature is constructed for each sensor node by combining the prediction compensation estimations with its own innovation measurements. Subsequently, a locally optimal distributed filtering scheme is presented, where the desirable time-varying filter gain matrix is expressed based on the solutions to certain matrix difference equations and a minimal upper bound matrix of the estimation error covariance is obtained at every sampling step. Compared with the existing results, the following challenges should be handled: 1) How to examine both the communication delays and the missing measurements within a unified framework? 2) How to actively compensate and reflect the available information of the mentioned phenomena in the distributed estimation algorithm? 3) How to depict the performance of the estimation algorithm from the theoretical aspects. The main contributions and advantages of this article could be outlined as: 1) the communication delays among the node transmissions are taken into account; 2) a prediction-based estimation updating rule is given to actively compensate the impacts caused by the communication delays during the state estimation exchanges among the nodes; 3) a new distributed filtering strategy with easy-to-implement form is provided via the active delay compensation of the state estimation prediction and the occurrence probabilities of missing measurements; and 4) a rigorously theoretical proof is presented to show the monotonicity between the estimation error covariance and the missing measurements. Finally, some simulations with comparative results are utilized to show the advantages of the prediction-based distributed filtering method proposed in this article.

The remainder of this article are outlined as follows. In Section II, both the mathematical model of the target plant and the related information of sensor networks are first provided. Moreover, the prediction compensation idea is introduced for the communication delays and a new distributed 
filter is constructed. The design of the prediction-based distributed filtering algorithm is provided in Section III and the desired expression form of the distributed filter gain is also presented. In Section IV, the mathematical analysis of the algorithm performance is conducted. In Section V, the simulation experiments are conducted to demonstrate the usefulness and differences of the new distributed filtering scheme based on the prediction compensation mechanism. Finally, some conclusion discussions are provided in Section VI.

Notations: $\mathscr{R}^{n_{x}}$ depicts the $n_{x}$-dimensional Euclidean space. $\mathbb{E}\{\cdot\}$ means the mathematical expectation of ".". $\operatorname{tr}(A)$ represents the trace of the matrix $A . A^{\mathrm{T}}$ and $A^{-1}$ denote the transpose of the matrix $A$ and the inverse of the matrix $A$, respectively. $\hat{x}_{j, k \mid k-\tau_{i j}}$ stands for the state prediction at the time step $k$ based on the delayed estimation $\hat{x}_{j, k-\tau_{i j}}$. The graph $\mathcal{G}=(\mathscr{V}, \mathscr{E}, \mathscr{H})$ is used to represent the topological structure with the node set $\mathscr{V}=\{1,2, \ldots, N\}$, the edge set $\mathscr{E} \subset \mathscr{V} \times \mathscr{V}$ and the weighted adjacency matrix $\mathscr{H}=\left[h_{i j}\right]_{N \times N}$.

\section{Problem Statements}

In this section, the prediction-based distributed filtering problem is formulated for addressed discrete time-varying nonlinear stochastic systems subject to communication delays and missing measurements, where the information is communicated via the sensor networks with $N$ sensor nodes. First, the related information of the sensor networks and the target plant is provided. Second, a new distributed filter is constructed in terms of the prediction mechanism for each sensor node, which involves the state estimations updated by predictive way and the related measurement innovations. In addition, the aims of the design problem of distributed filter based on the predictive compensation scheme are outlined.

\section{A. Sensor Networks and Target Plant}

In this section, both the related information of sensor networks and the addressed time-varying nonlinear target plant are introduced.

The network topological structure is characterized by the directed graph $\mathcal{G}=(\mathscr{V}, \mathscr{E}, \mathscr{H})$, where $\mathscr{V}=\{1,2, \ldots, N\}$ depicts the set of sensor nodes, $\mathscr{E} \subset \mathscr{V} \times \mathscr{V}$ stands for the edge set, and $\mathscr{H}=\left[h_{i j}\right]_{N \times N}$ represents the weighted adjacency matrix with the element $h_{i j}$ satisfying $h_{i j}>0 \Leftrightarrow(i, j) \in \mathscr{E}$. In particular, assume that $h_{i i}=1$. Moreover, the set of neighbors of sensor node $i$ is denoted by $\mathcal{N}_{i}$ with $\mathcal{N}_{i} \triangleq\{j \in \mathscr{V} \mid(i, j) \in$ $\mathscr{E}\} \backslash\{i\}$.

In this article, the mathematical model of addressed timevarying nonlinear multisensor systems is given as follows:

$$
\begin{aligned}
x_{k+1} & =F_{k} x_{k}+f_{k}\left(\xi_{k}, x_{k}\right)+B_{k} \varpi_{k} \\
y_{i, k} & =\varrho_{i, k} H_{i, k} x_{k}+v_{i, k}
\end{aligned}
$$

where $x_{k} \in \mathscr{R}^{n_{x}}$ is the state vector, and $y_{i, k} \in \mathscr{R}^{m_{y}}$ represents the measurement output collected by the $i$ th $(i=1,2, \ldots, N)$ sensor node. $\xi_{k}$ is a zero-mean white noise with unity variance. The process noise $\varpi_{k}$ and measurement noise $v_{i, k}$ have zero mean values, and their covariances are denoted by $W_{k}>0$ and $V_{i, k}>0$, respectively. $F_{k}, H_{i, k}(i=1,2, \ldots, N)$ and $B_{k}$ are known properly dimensional system matrices.
The stochastic nonlinearity $f_{k}\left(\xi_{k}, x_{k}\right)$ with $f_{k}\left(\xi_{k}, 0\right)=0$ satisfies

$$
\mathbb{E}\left\{f_{k}\left(\xi_{k}, x_{k}\right) \mid x_{k}\right\}=0
$$

and

$$
\begin{aligned}
\mathbb{E}\left\{f_{k}\left(\xi_{k}, x_{k}\right) f_{m}^{\mathrm{T}}\left(\xi_{m}, x_{m}\right) \mid x_{k}\right\} & =0, \quad k \neq m \\
\mathbb{E}\left\{f_{k}\left(\xi_{k}, x_{k}\right) f_{k}^{\mathrm{T}}\left(\xi_{k}, x_{k}\right) \mid x_{k}\right\} & =\sum_{i=1}^{t} \Gamma_{i, k} x_{k}^{\mathrm{T}} \Phi_{i, k} x_{k}
\end{aligned}
$$

where $t>0$ is a known integer, $\Gamma_{i, k}$ and $\Phi_{i, k}$ are properly dimensional known matrices.

The random variables $\varrho_{i, k}(i=1,2, \ldots, N)$ characterize the missing measurements and satisfy the following statistical property:

$$
\begin{aligned}
& \operatorname{Prob}\left\{\varrho_{i, k}=1\right\}=\mathbb{E}\left\{\varrho_{i, k}\right\}:=\bar{\varrho}_{i, k} \\
& \operatorname{Prob}\left\{\varrho_{i, k}=0\right\}=1-\mathbb{E}\left\{\varrho_{i, k}\right\}:=1-\bar{\varrho}_{i, k}
\end{aligned}
$$

where $\bar{\varrho}_{i, k} \in[0,1]$ are known constant scalars. Throughout this article, we suppose that $\xi_{k}, \varpi_{k}, v_{i, k}, \varrho_{i, k}$, and $x_{0}$ are mutually uncorrelated in $i$ and $k$.

\section{B. Design of Prediction-Based Distributed Filter}

Via the fixed topological structure, the following distributed filter is commonly constructed for the sensor node $i$ :

$$
\hat{x}_{i, k+1}=F_{k} \hat{x}_{i, k}+\mathscr{G}_{i, k}\left(y_{i, k}-\bar{\varrho}_{i, k} H_{i, k} \hat{x}_{i, k}\right)+\sum_{j \in \mathcal{N}_{i}} h_{i j}\left(\hat{x}_{i, k}-\hat{x}_{j, k}\right)
$$

with $\mathscr{G}_{i, k}$ being the distributed filter gain matrix to be designed. Notice that both the state estimations of adjacent nodes at current time step and the measurement innovations are needed. However, it should be pointed out that the state estimations exchanged by the connected sensor nodes might suffer from the network-induced communication delays. That is, the delayed estimation information can be available only after the network transmissions. Thus, the estimation performance would be deteriorated inevitably.

In what follows, the effects from the communication delays onto the estimation accuracy will be well examined. To begin, the communication delays are denoted by $\tau_{i j}$ between the sensor $i$ and sensor $j$. When designing the filter as in (5), the latest estimation $\hat{x}_{j, k-\tau_{i j}}$ is available at current sampling instant $k$ because of the communication delays. In order to improve the estimation accuracy and efficiency, the state estimations of adjacent nodes at current time step are predicted based on the delayed estimation information and the following prediction-based update method is adopted:

$$
\begin{aligned}
\hat{x}_{j, k-\tau_{i j}+1 \mid k-\tau_{i j}} & =F_{k-\tau_{i j}} \hat{x}_{j, k-\tau_{i j} \mid k-\tau_{i j}} \\
\hat{x}_{j, k-\tau_{i j}+2 \mid k-\tau_{i j}} & =F_{k-\tau_{i j}+1} \hat{x}_{j, k-\tau_{i j}+1 \mid k-\tau_{i j}} \\
& \vdots \\
\hat{x}_{j, k-1 \mid k-\tau_{i j}} & =F_{k-2} \hat{x}_{j, k-2 \mid k-\tau_{i j}} \\
\hat{x}_{j, k \mid k-\tau_{i j}} & =F_{k-1} \hat{x}_{j, k-1 \mid k-\tau_{i j}}
\end{aligned}
$$

where $\hat{x}_{j, k-\tau_{i j} \mid k-\tau_{i j}} \triangleq \hat{x}_{j, k-\tau_{i j}}$. Next, in order to facilitate further expression, we define $F_{k}^{\tau_{i j}}=\prod_{j=1}^{\tau_{i j}} F_{k-j}$. Then, it follows 
from the prediction idea in the equation (6) that $\hat{x}_{j, k \mid k-\tau_{i j}}=$ $F_{k}^{\tau_{i j}} \hat{x}_{j, k-\tau_{i j}}$.

Via the prediction estimation idea as in (6), the following distributed filtering scheme of the active compensation format is introduced:

$$
\begin{aligned}
\hat{x}_{i, k+1}= & F_{k} \hat{x}_{i, k}+\mathscr{G}_{i, k}\left(y_{i, k}-\bar{\varrho}_{i, k} H_{i, k} \hat{x}_{i, k}\right) \\
& +\sum_{j \in \mathcal{N}_{i}} h_{i j}\left(\hat{x}_{i, k}-\hat{x}_{j, k \mid k-\tau_{i j}}\right)
\end{aligned}
$$

with $\mathscr{G}_{i, k}$ being the desired filter parameter to be constructed.

Next, let $e_{i, k+1}=x_{k+1}-\hat{x}_{i, k+1}$ be the filtering error. Then, from (1) and (7), one has

$$
\begin{aligned}
e_{i, k+1}= & \left(F_{k}-\bar{\varrho}_{i, k} \mathscr{G}_{i, k} H_{i, k}\right) e_{i, k}+f_{k}\left(\xi_{k}, x_{k}\right) \\
& +B_{k} \bar{\varpi}_{k}-\tilde{\varrho}_{i, k} \mathscr{G}_{i, k} H_{i, k} x_{k}-\mathscr{G}_{i, k} v_{i, k} \\
& -\sum_{j \in \mathcal{N}_{i}} h_{i j}\left(\hat{x}_{i, k}-\hat{x}_{j, k \mid k-\tau_{i j}}\right)
\end{aligned}
$$

with $\tilde{\varrho}_{i, k}=\varrho_{i, k}-\bar{\varrho}_{i, k}$. In order to characterize the estimation accuracy, define the filtering error covariance as $\mathscr{X}_{i, k}=$ $\mathbb{E}\left\{e_{i, k} e_{i, k}^{\mathrm{T}}\right\}$.

Remark 1: So far, a new distributed filtering scheme under the active compensation method is given in (7), where both the prediction estimations of adjacent sensor nodes and its measurement innovation at the same sampling step are utilized during the design of the distributed filter. In particular, the communication delays $\tau_{i j}$ are considered in this article and the induced effects are compensated by utilizing the updating rule in (6). Accordingly, based on the delayed estimation $\hat{x}_{j, k-\tau_{i j}}$, the prediction estimation $\hat{x}_{j, k \mid k-\tau_{i j}}$ at the time step $k$ can be obtained and adopted in (7). In what follows, the validity of the prediction-based estimation approach will be shown, where the distributed filtering approach via the delay prediction compensation could provide better estimation accuracy than the one without the delay prediction compensation.

Now, we are ready to summarize the aims of this article, which include two aspects.

1) A new prediction-based distributed filtering algorithm with active compensation mechanism is developed, and the explicit form of the distributed filter parameter $\mathscr{G}_{i, k}$ is provided to minimize the upper bound matrix of the estimation error covariance.

2) The performance analysis problem of the developed distributed estimation algorithm is discussed, where the relationship between the missing probabilities and filtering algorithm accuracy is pointed out by providing the theoretical proof.

\section{Design of Prediction-Based Filtering Scheme}

In this section, the recursion expression of the estimation error covariance is provided in terms of the corresponding definition. Second, a minimized upper bound matrix of the estimation error covariance is found and the desirable filter parameter matrix is constructed accordingly. Third, the developed prediction-based suboptimal distributed filtering (PBSODF) algorithm is outlined to facilitate the implementations.
To begin, the following theorem presents the recursion expression of the resultant filtering error covariance.

Theorem 1: Consider the time-varying nonlinear stochastic system (1) and the distributed filter (7) based on the prediction compensation. The recursion expression of the filtering error covariance is described as

$$
\begin{aligned}
\mathscr{X}_{i, k+1}= & \left(F_{k}-\bar{\varrho}_{i, k} \mathscr{G}_{i, k} H_{i, k}\right) \mathscr{X}_{i, k}\left(F_{k}-\bar{\varrho}_{i, k} \mathscr{G}_{i, k} H_{i, k}\right)^{\mathrm{T}} \\
& -\left(F_{k}-\bar{\varrho}_{i, k} \mathscr{G}_{i, k} H_{i, k}\right) \mathbb{E}\left\{\sum_{j \in \mathcal{N}_{i}} h_{i j} e_{i, k}\right. \\
& \left.\times\left(\hat{x}_{i, k}-\hat{x}_{j, k \mid k-\tau_{i j}}\right)^{\mathrm{T}}\right\} \\
- & \mathbb{E}\left\{\sum_{j \in \mathcal{N}_{i}} h_{i j}\left(\hat{x}_{i, k}-\hat{x}_{j, k \mid k-\tau_{i j}}\right) e_{i, k}^{\mathrm{T}}\left(F_{k}-\bar{\varrho}_{i, k} \mathscr{G}_{i, k} H_{i, k}\right)^{\mathrm{T}}\right\} \\
& +\sum_{p=1}^{t} \Gamma_{p, k} \mathbb{E}\left\{x_{k}^{\mathrm{T}} \Phi_{p, k} x_{k}\right\}+B_{k} W_{k} B_{k}^{\mathrm{T}} \\
& +\bar{\varrho}_{i, k}\left(1-\bar{\varrho}_{i, k}\right) \mathbb{E}\left\{\mathscr{G}_{i, k} H_{i, k} x_{k} x_{k}^{\mathrm{T}} H_{i, k}^{\mathrm{T}} \mathscr{G}_{i, k}^{\mathrm{T}}\right\} \\
& +\sum_{j \in \mathcal{N}_{i}} \sum_{s \in \mathcal{N}_{i}} h_{i j} h_{i s}\left(\hat{x}_{i, k}-\hat{x}_{\left.j, k \mid k-\tau_{i j}\right)}\right. \\
& \times\left(\hat{x}_{i, k}-\hat{x}_{s, k \mid k-\tau_{i s}}\right)^{\mathrm{T}}+\mathscr{G}_{i, k} V_{i, k} \mathscr{G}_{i, k}^{\mathrm{T}} .
\end{aligned}
$$

Proof: Based on the filtering error in (8) and the corresponding definition of the filtering error covariance, the following equation is obtained:

$$
\begin{aligned}
\mathscr{X}_{i, k+1}= & \left(F_{k}-\bar{\varrho}_{i, k} \mathscr{G}_{i, k} H_{i, k}\right) \mathscr{X}_{i, k}\left(F_{k}-\bar{\varrho}_{i, k} \mathscr{G}_{i, k} H_{i, k}\right)^{\mathrm{T}} \\
& -\left(F_{k}-\bar{\varrho}_{i, k} \mathscr{G}_{i, k} H_{i, k}\right) \mathbb{E}\left\{\sum_{j \in \mathcal{N}_{i}} h_{i j} e_{i, k}\left(\hat{x}_{i, k}-\hat{x}_{\left.j, k \mid k-\tau_{i j}\right)}\right)\right\} \\
& -\mathbb{E}\left\{\sum_{j \in \mathcal{N}_{i}} h_{i j}\left(\hat{x}_{i, k}-\hat{x}_{j, k \mid k-\tau_{i j}}\right) e_{i, k}^{\mathrm{T}}\left(F_{k}-\bar{\varrho}_{i, k} \mathscr{G}_{i, k} H_{i, k}\right)^{\mathrm{T}}\right\} \\
& +\sum_{p=1}^{t} \Gamma_{p, k} \mathbb{E}\left\{x_{k}^{\mathrm{T}} \Phi_{p, k} x_{k}\right\}+B_{k} W_{k} B_{k}^{\mathrm{T}} \\
& +\bar{\varrho}_{i, k}\left(1-\bar{\varrho}_{i, k}\right) \mathbb{E}\left\{\mathscr{G}_{i, k} H_{i, k} x_{k} x_{k}^{\mathrm{T}} H_{i, k}^{\mathrm{T}} \mathscr{G}_{i, k}^{\mathrm{T}}\right\} \\
& +\sum_{j \in \mathcal{N}_{i}} \sum_{s \in \mathcal{N}_{i}} h_{i j} h_{i s}\left(\hat{x}_{i, k}-\hat{x}_{j, k \mid k-\tau_{i j}}\right)\left(\hat{x}_{i, k}-\hat{x}_{s, k \mid k-\tau_{i s}}\right)^{\mathrm{T}} \\
& +\mathscr{G}_{i, k} V_{i, k} \mathscr{G}_{i, k}^{\mathrm{T}}+\sum_{s=1}^{14}\left[\mathcal{A}_{i, k}^{s}+\left(\mathcal{A}_{i, k}^{s}\right)^{\mathrm{T}}\right]
\end{aligned}
$$

where

$$
\begin{aligned}
& \mathcal{A}_{i, k}^{1}=\mathbb{E}\left\{\left(F_{k}-\bar{\varrho}_{i, k} \mathscr{G}_{i, k} H_{i, k}\right) e_{i, k} f_{k}^{\mathrm{T}}\left(\xi_{k}, x_{k}\right)\right\} \\
& \mathcal{A}_{i, k}^{2}=\mathbb{E}\left\{\left(F_{k}-\bar{\varrho}_{i, k} \mathscr{G}_{i, k} H_{i, k}\right) e_{i, k} \varpi_{k}^{\mathrm{T}} B_{k}^{\mathrm{T}}\right\} \\
& \mathcal{A}_{i, k}^{3}=-\mathbb{E}\left\{\left(F_{k}-\bar{\varrho}_{i, k} \mathscr{G}_{i, k} H_{i, k}\right) \tilde{\varrho}_{i, k} e_{i, k} x_{k}^{\mathrm{T}} C_{i, k}^{\mathrm{T}} \mathscr{G}_{i, k}^{\mathrm{T}}\right\} \\
& \mathcal{A}_{i, k}^{4}=-\mathbb{E}\left\{\left(F_{k}-\bar{\varrho}_{i, k} \mathscr{G}_{i, k} H_{i, k}\right) e_{i, k} v_{i, k}^{\mathrm{T}} \mathscr{G}_{i, k}^{\mathrm{T}}\right\} \\
& \mathcal{A}_{i, k}^{5}=\mathbb{E}\left\{f_{k}\left(\xi_{k}, x_{k}\right) \varpi_{k}^{\mathrm{T}} B_{k}^{\mathrm{T}}\right\} \\
& \mathcal{A}_{i, k}^{6}=-\mathbb{E}\left\{\tilde{\varrho}_{i, k} f_{k}\left(\xi_{k}, x_{k}\right) x_{k}^{\mathrm{T}} C_{i, k}^{\mathrm{T}} \varphi_{i, k}^{\mathrm{T}}\right\} \\
& \mathcal{A}_{i, k}^{7}=-\mathbb{E}\left\{f_{k}\left(\xi_{k}, x_{k}\right) v_{i, k}^{\mathrm{T}} \mathscr{G}_{i, k}^{\mathrm{T}}\right\}
\end{aligned}
$$




$$
\begin{aligned}
\mathcal{A}_{i, k}^{8} & =-\mathbb{E}\left\{\sum_{j \in \mathcal{N}_{i}} h_{i j} f_{k}\left(\xi_{k}, x_{k}\right)\left(\hat{x}_{i, k}-\hat{x}_{j, k \mid k-\tau_{i j}}\right)^{\mathrm{T}}\right\} \\
\mathcal{A}_{i, k}^{9} & =-\mathbb{E}\left\{\tilde{\varrho}_{i, k} B_{k} \varpi_{k} x_{k}^{\mathrm{T}} C_{i, k}^{\mathrm{T}} \mathscr{G}_{i, k}^{\mathrm{T}}\right\} \\
\mathcal{A}_{i, k}^{10} & =-\mathbb{E}\left\{B_{k} \varpi_{k} v_{i, k}^{\mathrm{T}} \mathscr{G}_{i, k}^{\mathrm{T}}\right\} \\
\mathcal{A}_{i, k}^{11} & =-\mathbb{E}\left\{\sum_{j \in \mathcal{N}_{i}} h_{i j} B_{k} \varpi_{k}\left(\hat{x}_{i, k}-\hat{x}_{j, k \mid k-\tau_{i j}}\right)^{\mathrm{T}}\right\} \\
\mathcal{A}_{i, k}^{12}= & \mathbb{E}\left\{\tilde{\varrho}_{i, k} \mathscr{G}_{i, k} H_{i, k} x_{k} v_{i, k}^{\mathrm{T}} \mathscr{G}_{i, k}^{\mathrm{T}}\right\} \\
\mathcal{A}_{i, k}^{13}= & \sum_{j \in \mathcal{N}_{i}} h_{i j} \mathbb{E}\left\{\tilde{\varrho}_{i, k} \mathscr{G}_{i, k} H_{i, k} x_{k}\left(\hat{x}_{i, k}-\hat{x}_{j, k \mid k-\tau_{i j}}\right)^{\mathrm{T}}\right\} \\
\mathcal{A}_{i, k}^{14}= & \sum_{j \in \mathcal{N}_{i}} h_{i j} \mathbb{E}\left\{\mathscr{G}_{i, k} v_{i, k}\left(\hat{x}_{i, k}-\hat{x}_{j, k \mid k-\tau_{i j}}\right)^{\mathrm{T}}\right\} .
\end{aligned}
$$

Next, we can verify that the terms $\mathcal{A}_{i, k}^{s}(s=1,2, \ldots, 14)$ in (11) are zero terms since $\xi_{k}, \varpi_{k}, v_{i, k}$, and $\varrho_{i, k}$ are mutually uncorrelated in $i$ and $k$. Thus, (9) can be obtained readily.

Remark 2: It should be noticed that some unknown terms are involved in (9), thus the exact value of the filtering error covariance is unavailable. Therefore, additional effort will be devoted to handle those unknown term in terms of the matrix theory. Accordingly, a suboptimal estimation method is adopted, i.e., a minimized upper bound matrix of $\mathscr{X}_{i, k+1}$ is obtained by designing proper filter parameter via the completing square technique and mathematical induction approach.

Theorem 2: Consider the time-varying nonlinear stochastic system (1) and the distributed filter (7) based on the prediction compensation. Let $\sigma_{r, k}(r=1,2)$ be known positive constants. If the following matrix difference equation:

$$
\begin{aligned}
\Theta_{i, k+1}= & \left(1+\sigma_{1, k}\right)\left(F_{k}-\bar{\varrho}_{i, k} \mathscr{G}_{i, k} H_{i, k}\right) \\
& \times \Theta_{i, k}\left(F_{k}-\bar{\varrho}_{i, k} \mathscr{G}_{i, k} H_{i, k}\right)^{\mathrm{T}}+\sum_{p=1}^{t} \Gamma_{p, k} \operatorname{tr}\left(\Phi_{p, k} \Xi_{p, k}\right) \\
& +B_{k} W_{k} B_{k}^{\mathrm{T}}+\bar{\varrho}_{i, k}\left(1-\bar{\varrho}_{i, k}\right) \mathscr{G}_{i, k} H_{i, k} \Xi_{i, k} H_{i, k}^{\mathrm{T}} \mathscr{G}_{i, k}^{\mathrm{T}} \\
& +\mathscr{G}_{i, k} V_{i, k} \mathscr{G}_{i, k}^{\mathrm{T}}+\left(1+\sigma_{1, k}^{-1}\right) \sum_{j \in \mathcal{N}_{i}} \sum_{s \in \mathcal{N}_{i}} h_{i j} h_{i s} \\
& \times\left(\hat{x}_{i, k}-\hat{x}_{j, k \mid k-\tau_{i j}}\right)\left(\hat{x}_{i, k}-\hat{x}_{s, k \mid k-\tau_{i s}}\right)^{\mathrm{T}}
\end{aligned}
$$

under the initial condition $\mathscr{X}_{i, 0} \leq \Theta_{i, 0}$ has a solution $\Theta_{i, k}>0$, where

$$
\Xi_{i, k}=\left(1+\sigma_{2, k}\right) \Theta_{i, k}+\left(1+\sigma_{2, k}^{-1}\right) \hat{x}_{i, k} \hat{x}_{i, k}^{\mathrm{T}}
$$

then we can testify that

$$
\mathscr{X}_{i, k+1} \leq \Theta_{i, k+1} .
$$

Furthermore, if we adopt the following filter gain matrix:

$$
\begin{aligned}
\mathscr{G}_{i, k}=\left(1+\sigma_{1, k}\right) \bar{\varrho}_{i, k} F_{k} \Theta_{i, k} H_{i, k}^{\mathrm{T}} \\
\times\left[\left(1+\sigma_{1, k}\right) \bar{\varrho}_{i, k}^{2} H_{i, k} \Theta_{i, k} H_{i, k}^{\mathrm{T}}+\bar{\varrho}_{i, k}\left(1-\bar{\varrho}_{i, k}\right)\right. \\
\left.\quad \times H_{i, k} \Xi_{i, k} H_{i, k}^{\mathrm{T}}+V_{i, k}\right]^{-1}
\end{aligned}
$$

it is observed that the minimized upper bound matrix $\Theta_{i, k+1}$ can be described by

$$
\begin{aligned}
\Theta_{i, k+1}= & \left(1+\sigma_{1, k}\right) F_{k} \Theta_{i, k} F_{k}^{\mathrm{T}}+B_{k} W_{k} B_{k}^{\mathrm{T}}+\left(1+\sigma_{1, k}^{-1}\right) \\
& \times \sum_{j \in \mathcal{N}_{i}} \sum_{s \in \mathcal{N}_{i}} h_{i j} h_{i s}\left(\hat{x}_{i, k}-\hat{x}_{j, k \mid k-\tau_{i j}}\right)\left(\hat{x}_{i, k}-\hat{x}_{s, k \mid k-\tau_{i s}}\right)^{\mathrm{T}} \\
- & \left(1+\sigma_{1, k}\right)^{2} \bar{\varrho}_{i, k}^{2} F_{k} \Theta_{i, k} H_{i, k}^{\mathrm{T}} \\
\times & {\left[\left(1+\sigma_{1, k}\right) \bar{\varrho}_{i, k}^{2} H_{i, k} \Theta_{i, k} H_{i, k}^{\mathrm{T}}+\bar{\varrho}_{i, k}\left(1-\bar{\varrho}_{i, k}\right)\right.} \\
& \left.\times H_{i, k} \Xi_{i, k} H_{i, k}^{\mathrm{T}}+V_{i, k}\right]^{-1} H_{i, k} \Theta_{i, k}^{\mathrm{T}} F_{k}^{\mathrm{T}} .
\end{aligned}
$$

Proof: By employing the completing square technique and the mathematical induction method, the assertions in this theorem can be verified. First, suppose that $\mathscr{X}_{i, k} \leq \Theta_{i, k}$ holds. Second, there is a need to show that $\mathscr{X}_{i, k+1} \leq \Theta_{i, k+1}$.

Now, the involved cross-terms in (9) are first tackled. It follows from simple computations that the following inequality can be obtained:

$$
\begin{aligned}
& -\left(F_{k}-\bar{\varrho}_{i, k} \mathscr{G}_{i, k} H_{i, k}\right) \mathbb{E}\left\{\sum_{j \in \mathcal{N}_{i}} h_{i j} e_{i, k}\left(\hat{x}_{i, k}-\hat{x}_{\left.j, k \mid k-\tau_{i j}\right)}\right)\right\} \\
& -\mathbb{E}\left\{\sum_{j \in \mathcal{N}_{i}} h_{i j}\left(\hat{x}_{i, k}-\hat{x}_{\left.j, k \mid k-\tau_{i j}\right)}\right) e_{i, k}^{\mathrm{T}}\left(F_{k}-\bar{\varrho}_{i, k} \mathscr{G}_{i, k} H_{i, k}\right)^{\mathrm{T}}\right\} \\
& \leq \sigma_{1, k}\left(F_{k}-\bar{\varrho}_{i, k} \mathscr{G}_{i, k} H_{i, k}\right) \mathscr{X}_{i, k}\left(F_{k}-\bar{\varrho}_{i, k} \mathscr{G}_{i, k} H_{i, k}\right)^{\mathrm{T}} \\
& \quad+\sigma_{1, k}^{-1} \sum_{j \in \mathcal{N}_{i} s \in \mathcal{N}_{i}} \sum_{i j} h_{i s}\left(\hat{x}_{i, k}-\hat{x}_{j, k \mid k-\tau_{i j}}\right)\left(\hat{x}_{i, k}-\hat{x}_{s, k \mid k-\tau_{i s}}\right)^{\mathrm{T}}
\end{aligned}
$$

with $\sigma_{1, k}>0$ being the constant scalars. Besides, the following equations:

$$
\sum_{p=1}^{t} \Gamma_{p, k} \mathbb{E}\left\{x_{k}^{\mathrm{T}} \Phi_{p, k} x_{k}\right\}=\sum_{p=1}^{t} \Gamma_{p, k} \operatorname{tr}\left(\Phi_{p, k} \mathbb{E}\left\{x_{k} x_{k}^{\mathrm{T}}\right\}\right)
$$

are true. Moreover, it follows from $x_{k}=e_{i, k}+\hat{x}_{i, k}$ that:

$$
\begin{aligned}
\mathbb{E}\left\{x_{k} x_{k}^{\mathrm{T}}\right\} & \leq\left(1+\sigma_{2, k}\right) \mathscr{X}_{i, k}+\left(1+\sigma_{2, k}^{-1}\right) \hat{x}_{i, k} \hat{x}_{i, k}^{\mathrm{T}} \\
& \triangleq \bar{\Xi}_{i, k}
\end{aligned}
$$

with $\sigma_{2, k}$ being known positive constant scalars. Substituting (19) into (18) leads to

$$
\sum_{p=1}^{t} \Gamma_{p, k} \mathbb{E}\left\{x_{k}^{\mathrm{T}} \Phi_{p, k} x_{k}\right\} \leq \sum_{p=1}^{t} \Gamma_{p, k} \operatorname{tr}\left(\Phi_{p, k} \bar{\Xi}_{p, k}\right) .
$$

From (19), the sixth term in (9) obeys

$$
\begin{aligned}
& \bar{\varrho}_{i, k}\left(1-\bar{\varrho}_{i, k}\right) \mathbb{E}\left\{\mathscr{G}_{i, k} H_{i, k} x_{k} x_{k}^{\mathrm{T}} H_{i, k}^{\mathrm{T}} \mathscr{G}_{i, k}^{\mathrm{T}}\right\} \\
& \quad \leq \bar{\varrho}_{i, k}\left(1-\bar{\varrho}_{i, k}\right) \mathscr{G}_{i, k} H_{i, k} \bar{\Xi}_{i, k} H_{i, k}^{\mathrm{T}} \mathscr{G}_{i, k}^{\mathrm{T}} .
\end{aligned}
$$


Thus, together with (9), (17), (20), and (21), it can be shown that

$$
\begin{aligned}
\mathscr{X}_{i, k+1} \leq & \left(1+\sigma_{1, k}\right)\left(F_{k}-\bar{\varrho}_{i, k} \mathscr{G}_{i, k} H_{i, k}\right) \mathscr{X}_{i, k}\left(F_{k}-\bar{\varrho}_{i, k} \mathscr{G}_{i, k} H_{i, k}\right)^{\mathrm{T}} \\
& +\sum_{i=1}^{t} \Gamma_{i, k} \operatorname{tr}\left(\Phi_{i, k} \bar{\Xi}_{i, k}\right)+B_{k} W_{k} B_{k}^{\mathrm{T}} \\
& +\bar{\varrho}_{i, k}\left(1-\bar{\varrho}_{i, k}\right) \mathscr{G}_{i, k} H_{i, k} \bar{\Xi}_{i, k} H_{i, k}^{\mathrm{T}} \mathscr{G}_{i, k}^{\mathrm{T}} \\
& +\mathscr{G}_{i, k} V_{i, k} \mathscr{G}_{i, k}^{\mathrm{T}}+\left(1+\sigma_{1, k}^{-1}\right) \sum_{j \in \mathcal{N}_{i}} \sum_{s \in \mathcal{N}_{i}} h_{i j} h_{i s} \\
& \times\left(\hat{x}_{i, k}-\hat{x}_{j, k \mid k-\tau_{i j}}\right)\left(\hat{x}_{i, k}-\hat{x}_{\left.s, k \mid k-\tau_{i s}\right)^{\mathrm{T}} .}\right.
\end{aligned}
$$

Consequently, according to the mathematical induction approach, it is easy to show the following result:

$$
\mathscr{X}_{i, k+1} \leq \Theta_{i, k+1} .
$$

Finally, the minimum upper bound matrix $\Theta_{i, k+1}$ is determined by properly constructing the distributed filter parameter matrix $\mathscr{G}_{i, k}$. By employing the completing square technique, the upper bound matrix $\Theta_{i, k+1}$ can be rewritten as

$$
\begin{aligned}
& \Theta_{i, k+1}=\left[\mathscr{G}_{i, k}-\left(1+\sigma_{1, k}\right) \bar{\varrho}_{i, k} F_{k} \Theta_{i, k} H_{i, k}^{\mathrm{T}}\right. \\
& \times\left(\left(1+\sigma_{1, k}\right) \bar{\varrho}_{i, k}^{2} H_{i, k} \Theta_{i, k} H_{i, k}^{\mathrm{T}}+\bar{\varrho}_{i, k}\left(1-\bar{\varrho}_{i, k}\right)\right. \\
&\left.\left.\times H_{i, k} \Xi_{i, k} H_{i, k}^{\mathrm{T}}+V_{i, k}\right)^{-1}\right] \\
& \times\left(\left(1+\sigma_{1, k}\right) \bar{\varrho}_{i, k}^{2} H_{i, k} \Theta_{i, k} H_{i, k}^{\mathrm{T}}\right. \\
&\left.+\bar{\varrho}_{i, k}\left(1-\bar{\varrho}_{i, k}\right) H_{i, k} \Xi_{i, k} H_{i, k}^{\mathrm{T}}+V_{i, k}\right) \\
& \times {\left[\mathscr{G}_{i, k}-\left(1+\sigma_{1, k}\right) \bar{\varrho}_{i, k} F_{k} \Theta_{i, k} H_{i, k}^{\mathrm{T}}\right.} \\
& \times\left(\left(1+\sigma_{1, k}\right) \bar{\varrho}_{i, k}^{2} H_{i, k} \Theta_{i, k} H_{i, k}^{\mathrm{T}}\right. \\
&\left.\left.\quad+\bar{\varrho}_{i, k}\left(1-\bar{\varrho}_{i, k}\right) H_{i, k} \Xi_{i, k} H_{i, k}^{\mathrm{T}}+V_{i, k}\right)^{-1}\right]^{\mathrm{T}} \\
& \times\left(1+\sigma_{1, k}\right)^{2} \bar{\varrho}_{i, k}^{2} F_{k} \Theta_{i, k} H_{i, k}^{\mathrm{T}} \\
&\left.\quad \times H_{i, k} \Xi_{i, k} H_{i, k}^{\mathrm{T}}+\bar{\varrho}_{i, k}\right)^{-1} H_{i, k} \Theta_{i, k} F_{k}^{\mathrm{T}} \\
&+\left(1+\sigma_{1, k}\right) F_{k} \Theta_{i, k} F_{k}^{\mathrm{T}}+B_{k} W_{k} B_{k}^{\mathrm{T}}+\left(1+\sigma_{1, k}^{-1}\right) \\
& \times \sum_{j \in \mathcal{N}_{i}} \sum_{s \in \mathcal{N}_{i}} h_{i j} h_{i s}\left(\hat{x}_{i, k}-\hat{x}_{j, k \mid k-\tau_{i j}}\right)\left(\hat{x}_{i, k}-\hat{x}_{s, k \mid k-\tau_{i s}}\right)^{\mathrm{T}} .
\end{aligned}
$$

Then, it can be shown that the obtained upper bound matrix $\Theta_{i, k+1}$ of the filtering error covariance can be minimized by simply taking

$$
\begin{aligned}
\mathscr{G}_{i, k}=(1 & \left.+\sigma_{1, k}\right) \bar{\varrho}_{i, k} F_{k} \Theta_{i, k} H_{i, k}^{\mathrm{T}} \\
\times & {\left[\left(1+\sigma_{1, k}\right) \bar{\varrho}_{i, k}^{2} H_{i, k} \Theta_{i, k} H_{i, k}^{\mathrm{T}}+\bar{\varrho}_{i, k}\left(1-\bar{\varrho}_{i, k}\right)\right.} \\
& \left.\times H_{i, k} \Xi_{i, k} H_{i, k}^{\mathrm{T}}+V_{i, k}\right]^{-1} .
\end{aligned}
$$

\section{Algorithm 1 Algorithm PBSODF}

Step 1: Let $k=0$ and initialize the related values.

Step 2: Obtain the state estimations $\hat{x}_{k \mid k-\tau_{i j}}$ from (6).

Step 3: The filter parameter matrix $\mathscr{G}_{i, k}$ is determined via (15) for the $i$-th sensor node.

Step 4: Calculate the state estimation $\hat{x}_{i, k+1}$ in terms of (7).

Step 5: The upper bound matrix $\Theta_{i, k+1}$ is calculated by (12). Step 6: Set $k=k+1$ and then go back to Step 2.

Besides, the minimized upper bound matrix $\Theta_{i, k+1}$ is

$$
\begin{aligned}
\Theta_{i, k+1}= & \left(1+\sigma_{1, k}\right) F_{k} \Theta_{i, k} F_{k}^{\mathrm{T}}+B_{k} W_{k} B_{k}^{\mathrm{T}}+\left(1+\sigma_{1, k}^{-1}\right) \\
\times & \sum_{j \in \mathcal{N}_{i}} \sum_{s \in \mathcal{N}_{i}} h_{i j} h_{i s}\left(\hat{x}_{i, k}-\hat{x}_{j, k \mid k-\tau_{i j}}\right)\left(\hat{x}_{i, k}-\hat{x}_{s, k \mid k-\tau_{i s}}\right)^{\mathrm{T}} \\
- & \left(1+\sigma_{1, k}\right)^{2} \bar{\varrho}_{i, k}^{2} F_{k} \Theta_{i, k} H_{i, k}^{\mathrm{T}} \\
\times & {\left[\left(1+\sigma_{1, k}\right) \bar{\varrho}_{i, k}^{2} H_{i, k} \Theta_{i, k} H_{i, k}^{\mathrm{T}}+\bar{\varrho}_{i, k}\left(1-\bar{\varrho}_{i, k}\right)\right.} \\
& \left.\times H_{i, k} \Xi_{i, k} H_{i, k}^{\mathrm{T}}+V_{i, k}\right]^{-1} H_{i, k} \Theta_{i, k} F_{k}^{\mathrm{T}} .
\end{aligned}
$$

Therefore, the proof of this theorem is complete.

Based on the prediction-based distributed filter and the recursion expressions in Theorem 2, the following PBSODF algorithm can be given.

Remark 3: Up to now, the influences from the communication delays and the missing measurements onto the estimation error covariance have been addressed and a prediction-based distributed filtering method has been given. In particular, the delay compensation estimation based on the predictive updating rule and the occurrence probabilities of the missing measurements have been explicitly reflected in Theorem 2 . The reason is that the communication delays $\tau_{i j}$ have been utilized in (6) at the prediction step and the occurrence probabilities $\bar{\varrho}_{i, k}$ have been adopted during the design of distributed filter (7). Compared with the existing estimation methods, we have made one of the first attempts to discuss the effects of communication delays among the adjacent node's communications. Besides, the time-varying characteristic of addressed dynamical networks has been well discussed and an appealing prediction-based estimation algorithm suitable for online computations has been developed accordingly. During the distributed filtering algorithm, the major step is to obtain the prediction estimation in Step 2 based on the updating rule (6) and have the filter parameter matrix $\mathscr{G}_{i, k}$ recursively in Step 3 via (15).

\section{Performance Analysis of PBSODF ALGORITHM}

In this section, the theoretical derivations are given to discuss the performance analysis problem of proposed PBSODF algorithm, i.e., the monotonicity feature between $\operatorname{tr}\left(\Theta_{i, k}\right)$ and $\bar{\varrho}_{i, k}$ is shown.

Theorem 3: For the proposed prediction-based distributed filtering approach, it is shown that $\operatorname{tr}\left(\Theta_{i, k}\right)$ is nonincreasing if the missing probability $\bar{\varrho}_{i, k}$ increases. 
Proof: In terms of the minimized upper bound matrix in (16), we have

$$
\begin{aligned}
\frac{\operatorname{dtr}\left(\Theta_{i, k}\right)}{\mathrm{d} \bar{\varrho}_{i, k}}=\operatorname{tr}\{ & -2\left(1+\sigma_{1, k}\right)^{2} \bar{\varrho}_{i, k} F_{k} \Theta_{i, k} H_{i, k}^{\mathrm{T}} \\
\times & {\left[\left(1+\sigma_{1, k}\right) \bar{\varrho}_{i, k}^{2} H_{i, k} \Theta_{i, k} H_{i, k}^{\mathrm{T}}+\bar{\varrho}_{i, k}\left(1-\bar{\varrho}_{i, k}\right)\right.} \\
& \left.\times H_{i, k} \Xi_{i, k} H_{i, k}^{\mathrm{T}}+V_{i, k}\right]^{-1} H_{i, k} \Theta_{i, k} F_{k}^{\mathrm{T}} \\
+ & \left(1+\sigma_{1, k}\right)^{2} \bar{\varrho}_{i, k}^{2} F_{k} \Theta_{i, k} H_{i, k}^{\mathrm{T}} \\
\times & {\left[\left(1+\sigma_{1, k}\right) \bar{\varrho}_{i, k} H_{i, k} \Theta_{i, k} H_{i, k}^{\mathrm{T}}+\bar{\varrho}_{i, k}\left(1-\bar{\varrho}_{i, k}\right)\right.} \\
& \left.\times H_{i, k} \Xi_{i, k} H_{i, k}^{\mathrm{T}}+V_{i, k}\right]^{-1} \\
\times & {\left[2\left(1+\sigma_{1, k}\right) \bar{\varrho}_{i, k} H_{i, k} \Theta_{i, k} H_{i, k}^{\mathrm{T}}+H_{i, k} \Xi_{i, k} H_{i, k}^{\mathrm{T}}\right.} \\
& \left.\quad-2 \bar{\varrho}_{i, k} H_{i, k} \Xi_{i, k} H_{i, k}^{\mathrm{T}}\right] \\
\times & {\left[\left(1+\sigma_{1, k}\right) \bar{\varrho}_{i, k} H_{i, k} \Theta_{i, k} H_{i, k}^{\mathrm{T}}+\bar{\varrho}_{i, k}\left(1-\bar{\varrho}_{i, k}\right)\right.} \\
& \left.\left.\times H_{i, k} \Xi_{i, k} H_{i, k}^{\mathrm{T}}+V_{i, k}\right]^{-1} H_{i, k} \Theta_{i, k} F_{k}^{\mathrm{T}}\right\} .
\end{aligned}
$$

Next, by introducing the positive semi-definite term $2 \varrho_{i, k} V_{i, k}$ onto the right-hand side of the above equation, one has

$$
\begin{aligned}
& \frac{\operatorname{dtr}\left(\Theta_{i, k}\right)}{\mathrm{d} \bar{\varrho}_{i, k}} \\
& \leq \operatorname{tr}\left\{-2\left(1+\sigma_{1, k}\right)^{2} \bar{\varrho}_{i, k} F_{k} \Theta_{i, k} H_{i, k}^{\mathrm{T}}\right. \\
& \times\left[\left(1+\sigma_{1, k}\right) \bar{\varrho}_{i, k}^{2} \times H_{i, k} \Theta_{i, k} H_{i, k}^{\mathrm{T}}+\bar{\varrho}_{i, k}\left(1-\bar{\varrho}_{i, k}\right)\right. \\
& \left.\quad \times H_{i, k} \Xi_{i, k} H_{i, k}^{\mathrm{T}}+V_{i, k}\right]^{-1} \\
& \times H_{i, k} \Theta_{i, k} F_{k}^{\mathrm{T}}+\left(1+\sigma_{1, k}\right)^{2} F_{k} \Theta_{i, k} H_{i, k}^{\mathrm{T}} \\
& \times\left[\left(1+\sigma_{1, k}\right) \bar{\varrho}_{i, k} H_{i, k} \Theta_{i, k} H_{i, k}^{\mathrm{T}}+\bar{\varrho}_{i, k}\left(1-\bar{\varrho}_{i, k}\right)\right. \\
& \left.\quad \times H_{i, k} \Xi_{i, k} H_{i, k}^{\mathrm{T}}+V_{i, k}\right]^{-1} \\
& \times\left[2 \overline { \varrho } _ { i , k } \left(\left(1+\sigma_{1, k}\right) \bar{\varrho}_{i, k}^{2} H_{i, k} \Theta_{i, k} H_{i, k}^{\mathrm{T}}+\bar{\varrho}_{i, k}\left(1-\bar{\varrho}_{i, k}\right)\right.\right. \\
& \left.\left.\quad \times H_{i, k} \Xi_{i, k} H_{i, k}^{\mathrm{T}}+V_{i, k}\right)-\bar{\varrho}_{i, k}^{2} H_{i, k} \Xi_{i, k} H_{i, k}^{\mathrm{T}}\right] \\
& \times\left[\left(1+\sigma_{1, k}\right) \bar{\varrho}_{i, k} H_{i, k} \Theta_{i, k} H_{i, k}^{\mathrm{T}}+\bar{\varrho}_{i, k}\left(1-\bar{\varrho}_{i, k}\right)\right. \\
& \left.\left.\quad \times H_{i, k} \Xi_{i, k} H_{i, k}^{\mathrm{T}}+V_{i, k}\right]^{-1} H_{i, k} \Theta_{i, k} F_{k}^{\mathrm{T}}\right\}
\end{aligned}
$$$$
\leq 0 .
$$

Then, it follows from (27) that the proof of this theorem is complete.

Remark 4: In Theorem 3, the inherent relationship between the upper bound matrix of the filtering error covariance and the missing probabilities is pointed out by providing the theoretical analysis. To be more specific, it can be concluded from Theorem 3 that, the smaller the occurrence probabilities $\bar{\varrho}_{i, k}$, the bigger $\operatorname{tr}\left(\Theta_{i, k}\right)$, and then the worse the estimation accuracy of the developed PBSODF algorithm is obtained. The essential reason is that less measurements can be available in the filter side when $\bar{\varrho}_{i, k}$ is small, then the estimation accuracy of the developed PBSODF algorithm is degraded directly, which is consistent with the engineering insight and will also be illustrated later via the comparative simulations.

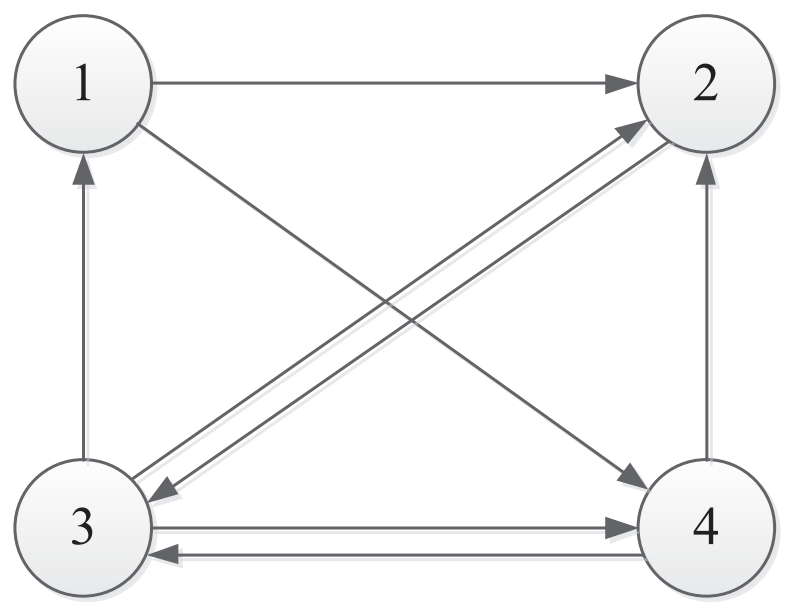

Fig. 1. Topological structure of the concerned sensor network.

\section{Comparative Simulations}

In this section, some comparative experiments are provided to further validate the advantages of the developed PBODF algorithm.

Consider the discrete nonlinear time-varying system (1) and the sensor network has four sensor nodes. The following system parameters are adopted:

$$
\begin{aligned}
F_{k} & =\left[\begin{array}{lll}
F_{k}^{11} & 0.35 & F_{k}^{13} \\
0.47 & 0.40 & 0.11 \\
0.37 & F_{k}^{32} & F_{k}^{33}
\end{array}\right] \\
B_{k} & =\left[\begin{array}{l}
0.14+0.03 \cos (3 k) \\
0.15+0.1 \sin (2 k) \\
0.18
\end{array}\right] \\
H_{1, k} & =\left[\begin{array}{lll}
1.4 & 0.77+0.1 \cos (k) & 1.01
\end{array}\right] \\
H_{2, k} & =\left[\begin{array}{lll}
1.6 & 0.76 & 0.9+0.12 \cos (7 k)
\end{array}\right] \\
H_{3, k} & =\left[\begin{array}{lll}
1.7 & 0.84 & 0.97
\end{array}\right], \\
H_{4, k} & =\left[\begin{array}{lll}
1.5+0.1 \sin (2 k) & 0.78 & 0.86
\end{array}\right] \\
F_{k}^{11} & =-0.8+0.3 \sin (k), F_{k}^{13}=0.27+0.41 \cos (2 k) \\
F_{k}^{32} & =0.52+0.01 \sin (k), F_{k}^{33}=0.62-0.05 \cos (3 k) .
\end{aligned}
$$

The variance of zero-mean process noise $w_{k}$ is $W_{k}=2.5$, and the variances of zero-mean measurement noises $v_{i, k}$ are $V_{1, k}=V_{2, k}=2$, and $V_{3, k}=V_{4, k}=1.5$, respectively.

Fig. 1 depicts the topological structure of concerned sensor network and the corresponding weighted adjacency matrix is described by

$$
\mathscr{H}=\left[h_{i j}\right]_{N \times N}=\left[\begin{array}{cccc}
1 & 0.07 & 0 & 0.17 \\
0 & 1 & 0.13 & 0 \\
0.07 & 0.094 & 1 & 0.076 \\
0 & 0.15 & 0.05 & 1
\end{array}\right] .
$$

The following stochastic nonlinear function $f\left(\xi_{k}, x_{k}\right)$ is considered:

$$
\begin{aligned}
& f\left(\xi_{k}, x_{k}\right)=\left[\begin{array}{l}
0.4 \\
0.5 \\
0.1
\end{array}\right]\left[0.5 \operatorname{sign}\left(x_{k}^{1}\right) x_{k}^{1} \xi_{k}^{1}+0.2 \operatorname{sign}\left(x_{k}^{2}\right)\right. \\
& \left.\times x_{k}^{2} \xi_{k}^{2}+0.4 \operatorname{sign}\left(x_{k}^{3}\right) x_{k}^{3} \xi_{k}^{3}\right]
\end{aligned}
$$




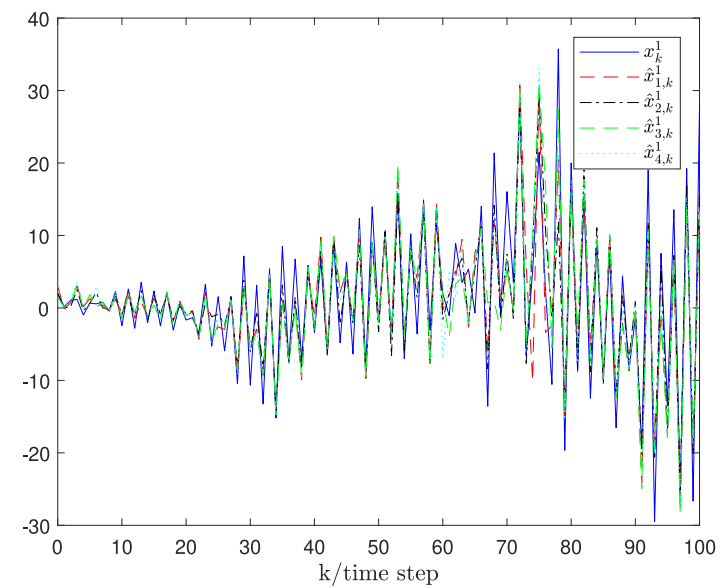

Fig. 2. Trajectories of $x_{k}^{1}$ and $\hat{x}_{i, k}^{1}$.

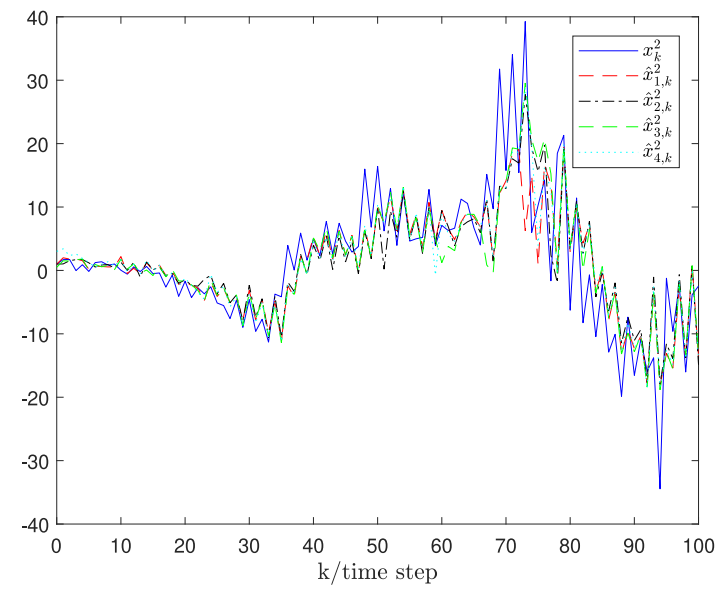

Fig. 3. Trajectories of $x_{k}^{2}$ and $\hat{x}_{i, k}^{2}$.

where $x_{k}^{p}$ is the $p$ th element of the target plant state, and $\xi_{k}^{p}$ $(p=1,2,3)$ is zero-mean white noise with unity covariance. Then, we can testify that

$$
\begin{aligned}
\mathbb{E}\left\{f\left(x_{k}, \xi_{k}\right) \mid x_{k}\right\}= & 0 \\
\mathbb{E}\left\{f\left(x_{k}, \xi_{k}\right) f^{\mathrm{T}}\left(x_{k}, \xi_{k}\right) \mid x_{k}\right\}= & {\left[\begin{array}{l}
0.4 \\
0.5 \\
0.1
\end{array}\right]\left[\begin{array}{c}
0.4 \\
0.5 \\
0.1
\end{array}\right]^{\mathrm{T}} } \\
& \times x_{k}^{\mathrm{T}}\left[\begin{array}{ccc}
0.25 & 0 & 0 \\
0 & 0.04 & 0 \\
0 & 0 & 0.16
\end{array}\right] x_{k} .
\end{aligned}
$$

For the purpose of simulation, select the initial values as $\bar{x}_{0}=\left[\begin{array}{lll}2 & 1 & 3\end{array}\right]^{\mathrm{T}}, \hat{x}_{1,0}=\left[\begin{array}{lll}3 & 1 & 3\end{array}\right]^{\mathrm{T}}, \hat{x}_{2,0}=\left[\begin{array}{lll}2 & 1 & 2\end{array}\right]^{\mathrm{T}}$, $\hat{x}_{3,0}=\left[\begin{array}{lll}1.5 & 0.5 & 4\end{array}\right]^{\mathrm{T}}, \hat{x}_{4,0}=\left[\begin{array}{lll}3 & 3 & 2\end{array}\right]^{\mathrm{T}}$, and $\hat{x}_{i, k}=$ $\left[\begin{array}{lll}0 & 0 & 0\end{array}\right]^{\mathrm{T}}(i=1,2,3,4, k<0)$. Moreover, assume that the communication delays are $\tau_{i j}=5$, the other parameters are given by $\sigma_{1, k}=0.1, \sigma_{2, k}=0.3$, and $\bar{\varrho}_{i, k}=0.95$. Then, the developed PBODF algorithm can be implemented and the corresponding simulations can be plotted in Figs. 2-8, where the actual state, estimated state, mean-square error (MSE) and the trace of upper bound matrix are depicted. For more details, Figs. 2-4 plot the actual system states and estimations by utilizing the PBODF method. Moreover, Figs. 5-8 provide the

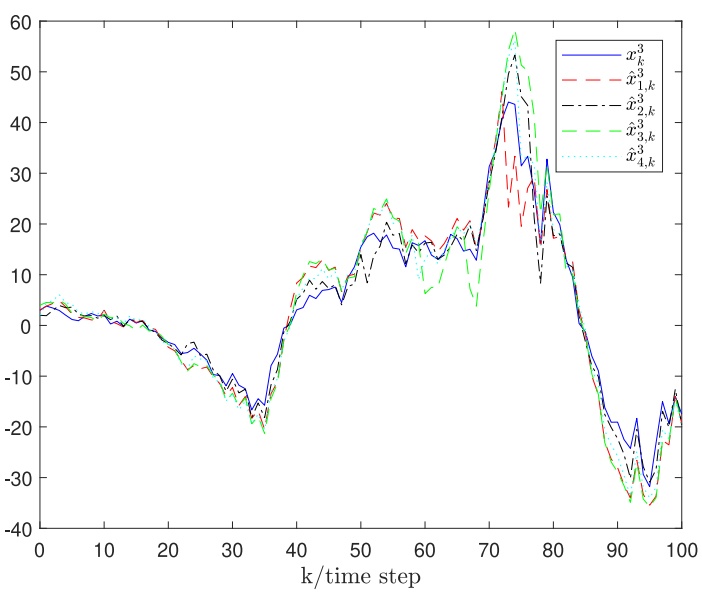

Fig. 4. Trajectories of $x_{k}^{3}$ and $\hat{x}_{i, k}^{3}$.

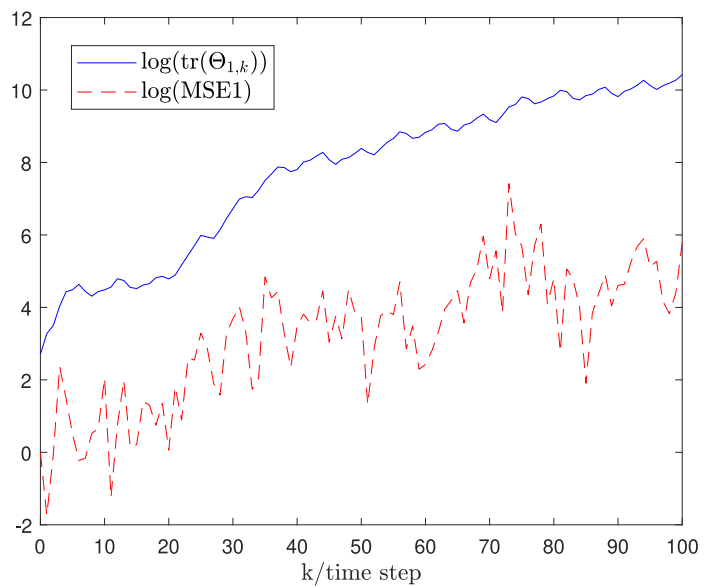

Fig. 5. $\log (\mathrm{MSE} 1)$ and the upper bound.

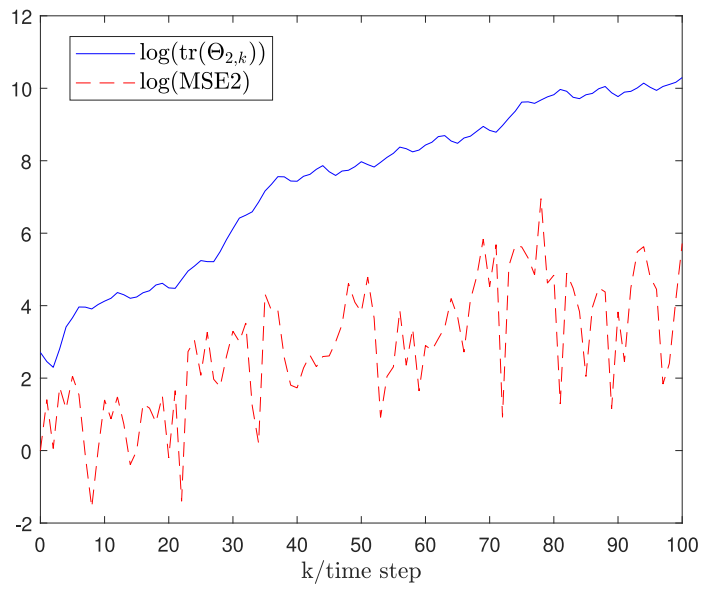

Fig. 6. Log(MSE2) and the upper bound.

$\log$ (MSE) based on the observations from four sensor nodes and the related upper bounds, which show the feasibility of the presented PBODF algorithm.

In order to further show the advantages of the newly prediction-based estimation approach, the effects of the communication delays between the node's transmissions are discussed and some comparative simulations are provided. In 


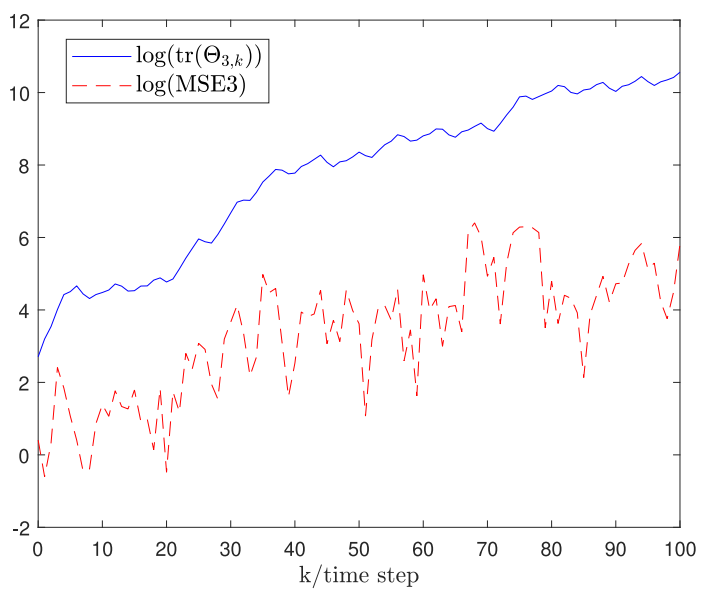

Fig. 7. Log(MSE3) and the upper bound.

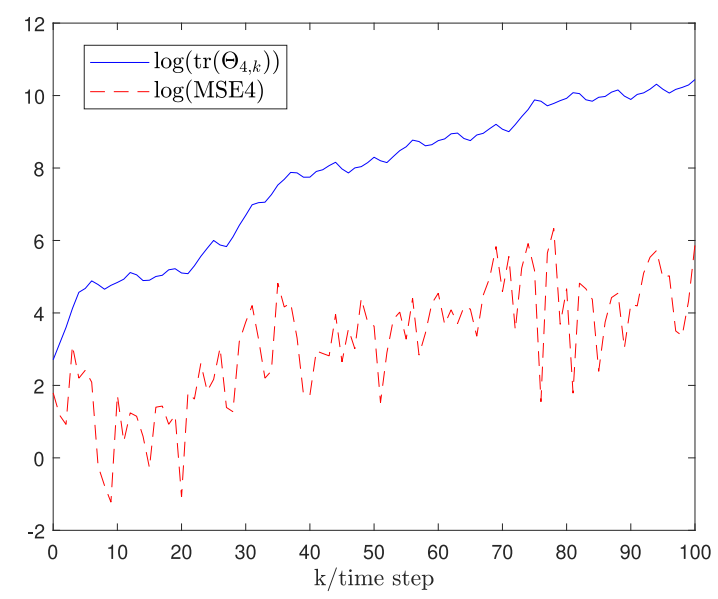

Fig. 8. Log(MSE4) and the upper bound.

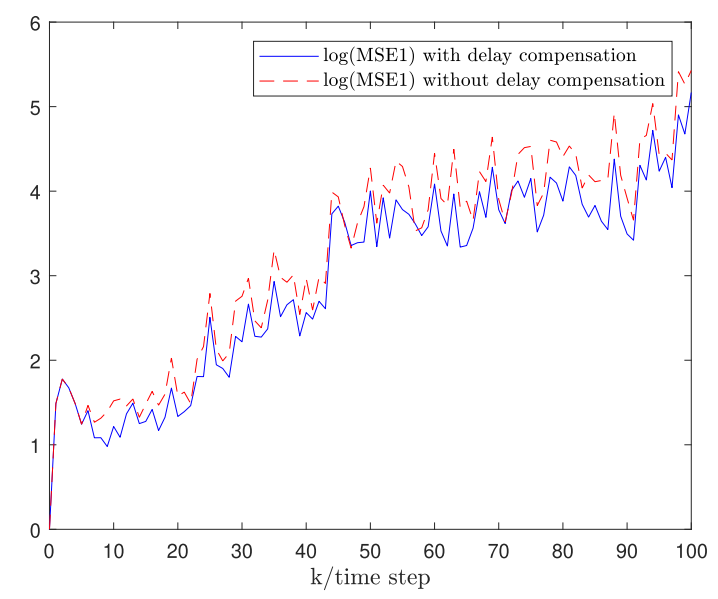

Fig. 9. Comparisons of $\log (\mathrm{MSE} 1)$.

particular, Figs. 9-12 plot the $\log$ (MSEs) with and without prediction compensation of the delayed estimation, where 500 simulation tests are made. It can be observed from Figs. 9-12 that the $\log$ (MSEs) are indeed below their upper bounds since the delay effects are clearly discussed and the delay compensations are utilized via the prediction updating rule proposed in this article. Then, it follows from the trajectories under the

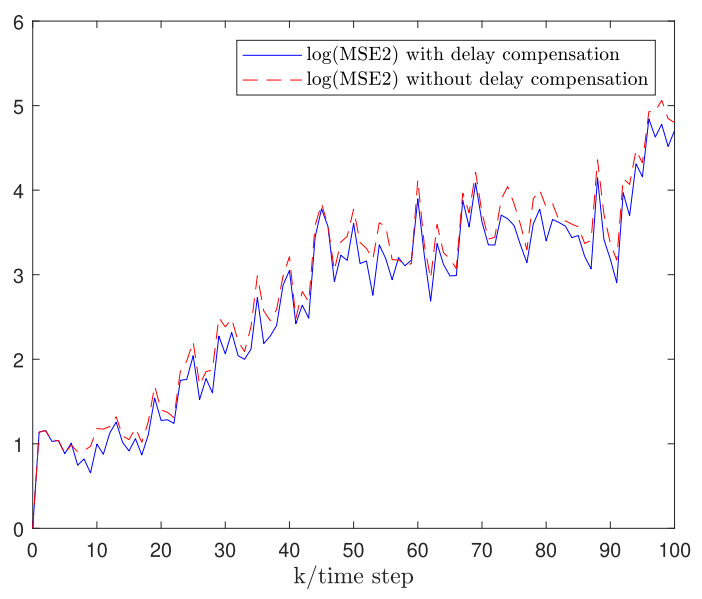

Fig. 10. Comparisons of $\log (\mathrm{MSE} 2)$.

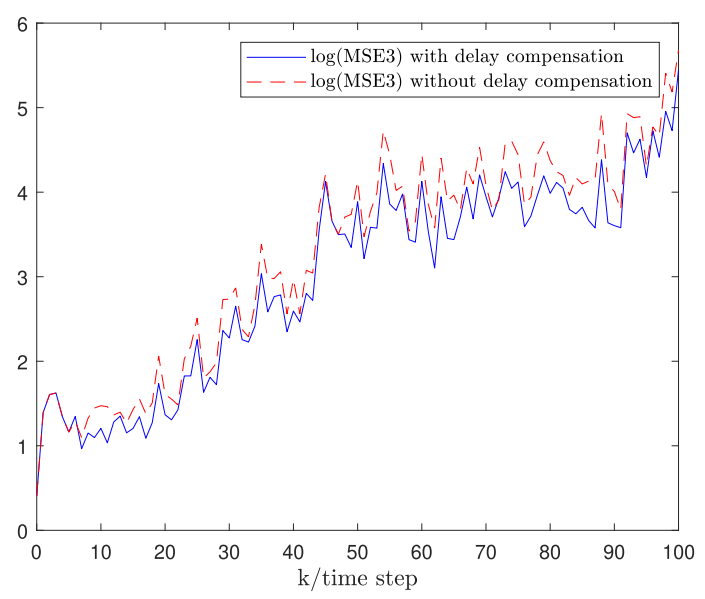

Fig. 11. Comparisons of $\log (\mathrm{MSE} 3)$.

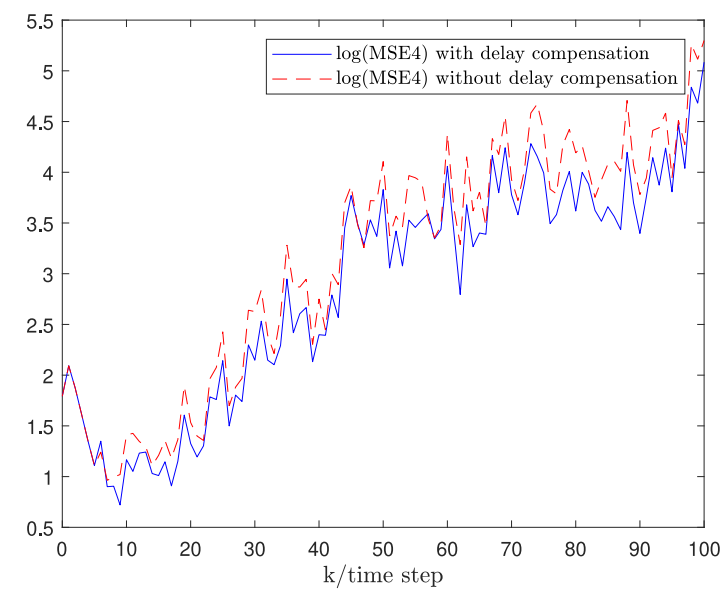

Fig. 12. Comparisons of $\log (\mathrm{MSE} 4)$.

comparative cases that the estimation accuracy of the proposed PBSODF algorithm is better than the one without prediction.

Besides, we are in a position to examine the corresponding changes of the filtering algorithm accuracy with respect to different missing probabilities. In particular, the relationships between the probabilities $\left(\bar{\varrho}_{i, k}=0.75,0.85,0.95,1\right)$ and the upper bound matrices are illustrated in Figs. 13-16. 


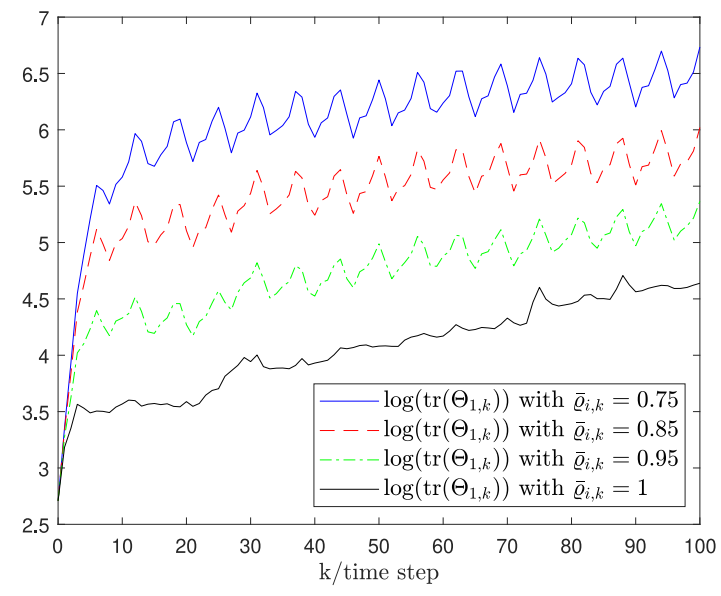

Fig. 13. $\log \left(\operatorname{tr}\left(\Theta_{1, k}\right)\right)$ regarding different missing probabilities.

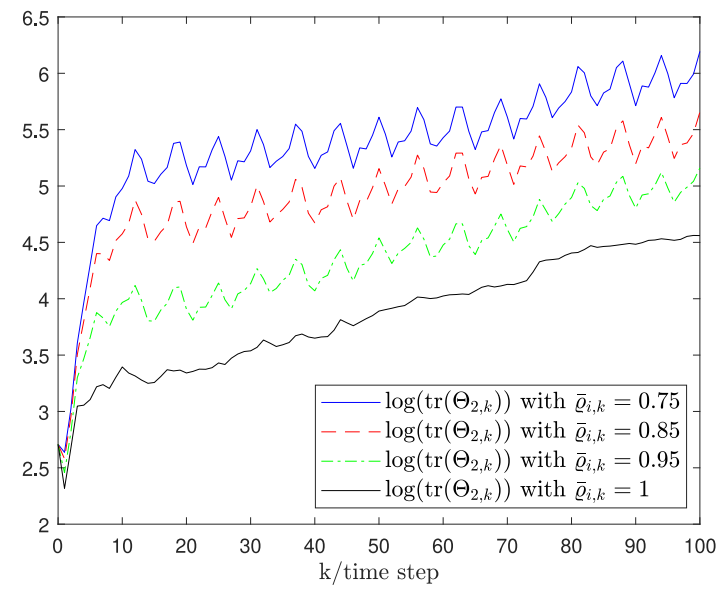

Fig. 14. $\log \left(\operatorname{tr}\left(\Theta_{2, k}\right)\right)$ regarding different missing probabilities.

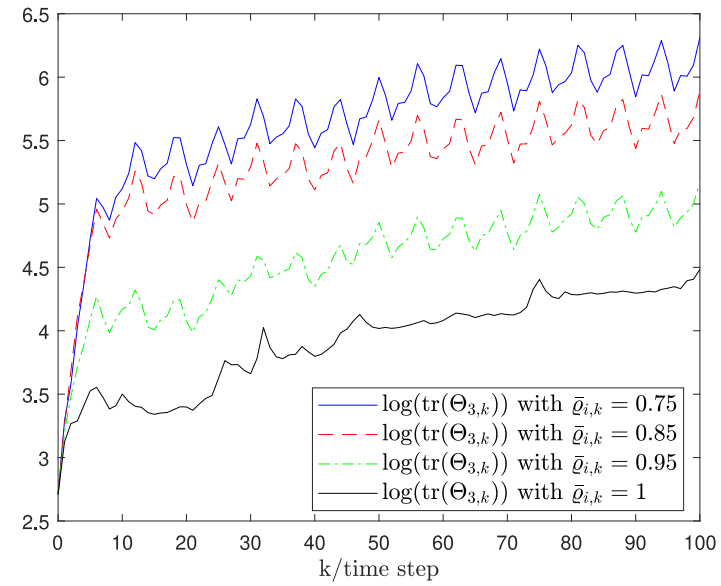

Fig. 15. $\log \left(\operatorname{tr}\left(\Theta_{3, k}\right)\right)$ regarding different missing probabilities.

From the simulations, we can see that the bigger the occurrence probability $\bar{\varrho}_{i, k}$, the more real measurements $y_{i, k}$ and the smaller $\operatorname{tr}\left(\Theta_{i, k}\right)$, and then the better estimation accuracy of the developed PBSODF algorithm is ensured, which are indeed consistent with the assertion in Theorem 3. Consequently, according to the above comparative simulations, it is observed

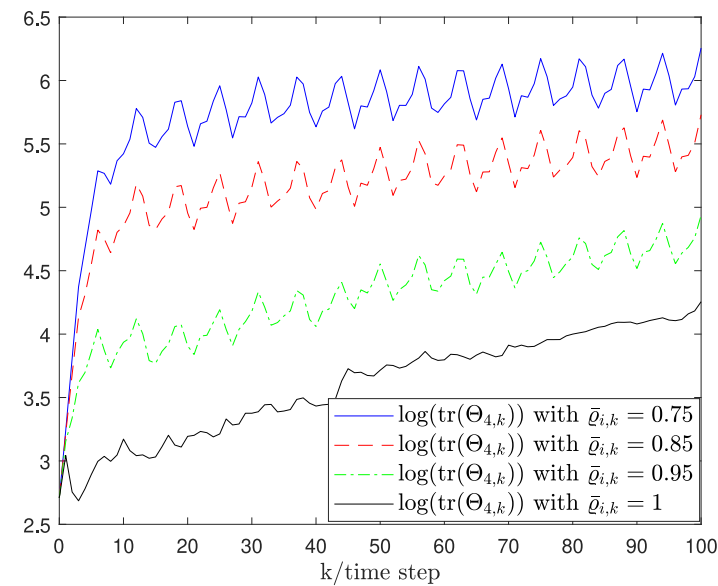

Fig. 16. $\log \left(\operatorname{tr}\left(\Theta_{4, k}\right)\right)$ regarding different missing probabilities.

that the new PBSODF algorithm performs a satisfactory estimation performance when the measurements/transmissions undergo the communication delays and missing measurements. The major reason lies in that the proposed PBSODF algorithm can actively compensate the influences from the communication delays and the missing measurements onto the filtering algorithm performance.

Remark 5: Up to now, the advantages of the newly proposed PBSODF algorithm has been shown by using the comparative simulations. Compared with the existing distributed filtering methods, the PBSODF algorithm has wider application domain since the communication delays among the node transmissions have been considered and compensated actively.

\section{CONCLUSION}

In this article, the prediction-based distributed filtering problem has been investigated for a class of discrete timevarying nonlinear stochastic systems with communication delays and missing measurements through sensor networks. A new distributed filtering strategy has been presented by fully taking both the prediction estimations and its own measurement innovation into consideration. Moreover, the algorithm performance evaluation problem has been carried out and the theoretical proof has been presented. It should be noted that the major features of the proposed method lies in that a hybrid distributed estimation algorithm has been proposed, which has active compensation ability. Moreover, the rigorous mathematical proof has been provided to show the monotonicity with regards to the distributed filtering algorithm performance. Further extensions include the design of a prediction-based distributed filtering scheme for more general systems with communication transmission protocols as in [23] and [45].

\section{REFERENCES}

[1] A. Abdelgawad, "Distributed Kalman filter with fast consensus for wireless sensor networks," Int. J. Wireless Inf. Netw., vol. 23, no. 1 , pp. 82-88, 2016.

[2] M. V. Basin, P. Shi, and P. Soto, "Central suboptimal $H_{\infty}$ filtering for nonlinear polynomial systems with multiplicative noise," J. Franklin Inst., vol. 347, no. 9, pp. 1740-1754, 2010. 
[3] R. Caballero-Águila, A. Hermoso-Carazo, J. Linares-Ṕerez, and Z. Wang, "A new approach to distributed fusion filtering for networked systems with random parameter matrices and correlated noises," Inf. Fusion, vol. 45, pp. 324-332, Jan. 2019.

[4] R. Caballero-Águila, A. Hermoso-Carazo, and J. Linares-Ṕerez, "Networked distributed fusion estimation under uncertain outputs with random transmission delays, packet losses and multi-packet processing," Signal Process., vol. 156, pp. 71-83, Mar. 2019.

[5] J. Chen, C. K. Ahn, H. R. Karim, J. Cao, and W. Qin, "An eventbased asynchronous approach to Markov jump systems with hidden mode detections and missing measurements," IEEE Trans. Syst., Man, Cybern., Syst., vol. 49, no. 9, pp. 1749-1758, Sep. 2019.

[6] W. Chen, D. Ding, H. Dong, and G. Wei, "Distributed resilient filtering for power systems subject to denial-of-service attacks," IEEE Trans. Syst., Man, Cybern., Syst., vol. 49, no. 8, pp. 1688-1697, Aug. 2019.

[7] D. Ding, Z. Wang, D. W. C. Ho, and G. Wei, "Distributed recursive filtering for stochastic systems under uniform quantizations and deception attacks through sensor networks," Automatica, vol. 78, pp. 231-240, Apr. 2017.

[8] H. Dong, Z. Wang, and H. Gao, "Distributed filtering for a class of time-varying systems over sensor networks with quantization errors and successive packet dropouts," IEEE Trans. Signal Process., vol. 60, no. 6, pp. 3164-3173, Jun. 2012.

[9] H. Dong, Z. Wang, and H. Gao, "Distributed $H_{\infty}$ filtering for a class of Markovian jump nonlinear time-delay systems over lossy sensor networks," IEEE Trans. Ind. Electron., vol. 60, no. 10, pp. 4665-4672, Oct. 2013.

[10] X. Ge and Q.-L. Han, "Distributed event-triggered $H_{\infty}$ filtering over sensor networks with communication delays," Inf. Sci., vol. 291, pp. 128-142, Jan. 2015

[11] X. Ge, Q.-L. Han, and Z. Wang, "A threshold-parameter-dependent approach to designing distributed event-triggered $H_{\infty}$ consensus filters over sensor networks," IEEE Trans. Cybern., vol. 49, no. 4, pp. 1148-1159, Apr. 2019.

[12] X. Ge, Q.-L. Han, X.-M. Zhang, L. Ding, and F. Yang, "Distributed event-triggered estimation over sensor networks: A survey," IEEE Trans. Cybern., vol. 50, no. 3, pp. 1306-1320, 2020, doi: 10.1109/TCYB.2019.2917179.

[13] J. Hu, G.-P. Liu, Z. Wang, and H. Zhang, "Prediction-based optimal distributed filtering with communication delay over sensor networks," in Proc. 38th Chin. Control Conf., Guangzhou, China, Jul. 2019, pp. 5315-5320.

[14] J. Hu, Z. Wang, and H. Gao, "Joint state and fault estimation for timevarying nonlinear systems with randomly occurring faults and sensor saturations," Automatica, vol. 97, pp. 150-160, Nov. 2018.

[15] J. Hu, Z. Wang, G.-P. Liu, and H. Zhang, "Variance-constrained recursive state estimation for time-varying complex networks with quantized measurements and uncertain inner coupling," IEEE Trans. Neural Netw. Learn. Syst., to be published, doi: 10.1109/TNNLS.2019.2927554.

[16] J. Hu, Z. Wang, S. Liu, and H. Gao, "A variance-constrained approach to recursive state estimation for time-varying complex networks with missing measurements," Automatica, vol. 64, pp. 155-162, 2016.

[17] J. Hu, H. Zhang, X. Yu, H. Liu, and D. Chen, "Design of sliding-modebased control for nonlinear systems with mixed-delays and packet losses under uncertain missing probability," IEEE Trans. Syst., Man, Cybern., Syst., to be published, doi: 10.1109/TSMC.2019.2919513.

[18] J. Hu, P. Zhang, Y. Kao, H. Liu, and D. Chen, "Sliding mode control for Markovian jump repeated scalar nonlinear systems with packet dropouts: The uncertain occurrence probabilities case," Appl. Math. Comput., vol. 362, 2019, Art. no. 124574, doi: 10.1016/j.amc.2019.124574.

[19] Y. Li, G.-P. Liu, S. Sun, and C. Tan, "Prediction-based approach to finite-time stabilization of networked control systems with time delays and data packet dropouts," Neurocomputing, vol. 329, pp. 320-328, Feb. 2019.

[20] Y.-J. Li, Z.-W. Huang, and J.-Z. Li, " $H_{\infty}$ state estimation for stochastic Markovian jumping neural network with time-varying delay and Leakage delay," Int. J. Autom. Comput., vol. 16, no. 3, pp. 329-340, 2019.

[21] G.-P. Liu, "Design and analysis of networked non-linear predictive control systems," IET Control Theory Appl., vol. 9, no. 11, pp. 1740-1745, 2015.

[22] G.-P. Liu, "Consensus and stability analysis of networked multiagent predictive control systems," IEEE Trans. Cybern., vol. 47, no. 4, pp. 1114-1119, Apr. 2017.

[23] K. Liu, E. Fridman, K. H. Johansson, and Y. Xia, "Quantized control under Round-Robin communication protocol," IEEE Trans. Ind. Electron., vol. 63, no. 7, pp. 4461-4471, Jul. 2016.
[24] K. Liu, H. Guo, Q. Zhang, and Y. Xia, "Distributed secure filtering for discrete-time systems under Round-Robin protocol and deception attacks," IEEE Trans. Cybern., to be published, doi: 10.1109/TCYB.2019.2897366.

[25] K. Liu, A. Selivanov, and E. Fridman, "Survey on time-delay approach to networked control," Annu. Rev. Control, vol. 48, pp. 57-79, 2019, doi: 10.1016/j.arcontrol.2019.06.005.

[26] Q. Liu, Z. Wang, X. He, and D. Zhou, "Event-based distributed filtering over Markovian switching topologies," IEEE Trans. Autom. Control, vol. 64 , no. 4, pp. 1595-1602, Apr. 2018.

[27] J. Ma and S. Sun, "Distributed fusion filter for networked stochastic uncertain systems with transmission delays and packet dropouts," Signal Process., vol. 130, pp. 268-278, Jan. 2017.

[28] J. Ma and S. Sun, "A general packet dropout compensation framework for optimal prior filter of networked multi-sensor systems," Inf. Fusion, vol. 45, pp. 128-137, Jan. 2019.

[29] M. Park, S.-H. Lee, O.-M. Kwon, and A. Seuret, "Closeness-centralitybased synchronization criteria for complex dynamical networks with interval time-varying coupling delays," IEEE Trans. Cybern., vol. 48, no. 7, pp. 2192-2202, Jul. 2018.

[30] W. Qian, Y. Gao, and Y. Yang, "Global consensus of multiagent systems with internal delays and communication delays," IEEE Trans. Syst., Man, Cybern., Syst., vol. 49, no. 10, pp. 1961-1970, Oct. 2019.

[31] G. G. Rigatos, "Derivative-free distributed filtering for MIMO robotic systems under delays and packet drops," Int. J. Adv. Robot. Syst., vol. 10, 2013, Art. no. 133.

[32] B. Shen, Z. Wang, and Y. S. Hung, "Distributed $H_{\infty}$-consensus filtering in sensor networks with multiple missing measurements: The finite-horizon case," Automatica, vol. 46, no. 10, pp. 1682-1688, 2010.

[33] I. Y. Song and V. Shin, "Distributed mixed continuous-discrete receding horizon filter for multisensory uncertain active suspension systems with measurement delays," IET Control Theory Appl., vol. 7, no. 15, pp. 1922-1931, 2013.

[34] O. Thapliyal, J. S. Nandiganahalli, and I. Hwang, "Kalman filtering with state-dependent packet losses," IET Control Theory Appl., vol. 13, no. 2, pp. 306-312, 2019.

[35] P. K. Varshney, "Multisensor data fusion," Electron. Commun. Eng. J., vol. 9, no. 6, pp. 245-253, 1997.

[36] X. Wang and G. Yang, "Event-triggered $H_{\infty}$ filtering for discretetime T-S fuzzy systems via network delay optimization technique," IEEE Trans. Syst., Man, Cybern., Syst., vol. 49, no. 10, pp. 2026-2035, Oct. 2019.

[37] C. Wen, Z. Wang, Q. Liu, and F. E. Alsaadi, "Recursive distributed filtering for a class of state-saturated systems with fading measurements and quantization effects," IEEE Trans. Syst., Man, Cybern., Syst., vol. 48 , no. 6, pp. 930-941, Jun. 2018.

[38] Y. Xu, T. Shen, X.-Y. Chen, L.-L. Bu, and N. Feng, "Predictive adaptive Kalman filter and its application to INS/UWB-integrated human localization with missing UWB-based measurements," Int. J. Autom. Comput., vol. 16, no. 5, pp. 604-613, 2019.

[39] H. Yan, P. Li, H. Zhang, X. Zhan, and F. Yang, "Event-triggered distributed fusion estimation of networked multisensor systems with limited information," IEEE Trans. Syst., Man, Cybern., Syst., to be published, doi: 10.1109/TSMC.2018.2874804.

[40] H. Yan, F. Qian, F. Yang, and H. Shi, " $H_{\infty}$ filtering for nonlinear networked systems with randomly occurring distributed delays, missing measurements and sensor saturation," Inf. Sci., vol. 370, pp. 772-782, Nov. 2016.

[41] H. Yan, Q. Yang, H. Zhang, F. Yang, and Z. Zhan, "Distributed $H_{\infty}$ state estimation for a class of filtering networks with time-varying switching topologies and packet losses," IEEE Trans. Syst., Man, Cybern., Syst., vol. 48, no. 12, pp. 2047-2057, Dec. 2018.

[42] X. Yin, Z. Li, L. Zhang, and M. Han, "Distributed state estimation of sensor-network systems subject to Markovian channel switching with application to a chemical process," IEEE Trans. Syst., Man, Cybern., Syst., vol. 48, no. 6, pp. 864-874, Jun. 2018.

[43] D. Zhang, Q.-L. Han, and X.-M. Zhang, "Network-based modeling and proportional-integral control for direct-drive-wheel systems in wireless network environments," IEEE Trans. Cybern., to be published, doi: 10.1109/TCYB.2019.2924450.

[44] D. Zhang, Z. Xu, H. R. Karimi, and Q.-G. Wang, "Distributed filtering for switched linear systems with sensor networks in presence of packet dropouts and quantization," IEEE Trans. Circuits Syst. I, Reg. Papers, vol. 64 , no. 10 , pp. $2783-2796$, Oct. 2017. 
[45] H. Zhang, Z. Wang, H. Yan, F. Yang, and X. Zhou, "Adaptive eventtriggered transmission scheme and $H_{\infty}$ filtering co-design over a filtering network with switching topology," IEEE Trans. Cybern., vol. 49, no. 12 , pp. 4296-4307, Dec. 2019.

[46] L. Zhang, Z. Ning, and Z. Wang, "Distributed filtering for fuzzy timedelay systems with packet dropouts and redundant channels," IEEE Trans. Syst., Man, Cybern., Syst., vol. 46, no. 4, pp. 559-572, Apr. 2016.

[47] W.-A. Zhang, G. Feng, and L. Yu, "Multi-rate distributed fusion estimation for sensor networks with packet losses," Automatica, vol. 48, no. 9 , pp. 2016-2028, 2012.

[48] Y. Zhang, F. Li, and Y. Chen, "Leader-following-based distributed Kalman filtering in sensor networks with communication delay," $J$. Franklin Inst., vol. 354, no. 16, pp. 7504-7520, 2017.

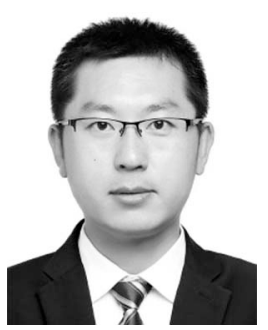

Jun Hu (Member, IEEE) received the B.Sc. degree in information and computation science and the M.Sc. degree in applied mathematics from the Harbin University of Science and Technology, Harbin, China, in 2006 and 2009, respectively, and the Ph.D. degree in control science and engineering from the Harbin Institute of Technology, Harbin, in 2013.

From September 2010 to September 2012, he was a visiting $\mathrm{Ph} . \mathrm{D}$. student with the Department of Information Systems and Computing, Brunel University London, Uxbridge, U.K. From May 2014 to April 2016, he was an Alexander von Humboldt Research Fellow with the University of Kaiserslautern, Kaiserslautern, Germany. He is with the Department of Mathematics, Harbin University of Science and Technology and also with the School of Engineering, University of South Wales, Pontypridd, U.K. He has published more than 50 papers in refereed international journals. His research interests include nonlinear control, filtering and fault estimation, time-varying systems, and complex networks.

Dr. Hu serves as a Reviewer for Mathematical Reviews, as an Editor for IEEE ACCESS, Neurocomputing, the Journal of Intelligent and Fuzzy Systems, Neural Processing Letters, Systems Science and Control Engineering, and as a Guest Editor for the International Journal of General Systems and Information Fusion.

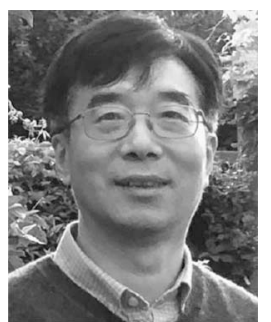

Zidong Wang (Fellow, IEEE) was born in Jiangsu, China, in 1966. He received the B.Sc. degree in mathematics from Suzhou University, Suzhou, China, in 1986, and the M.Sc. degree in applied mathematics and the Ph.D. degree in electrical engineering from the Nanjing University of Science and Technology, Nanjing, China, in 1990 and 1994 respectively.

$\mathrm{He}$ is currently a Professor of Dynamical Systems and Computing with the Department of Computer Science, Brunel University London, Uxbridge, U.K. From 1990 to 2002, he held teaching and research appointments in universities in China, Germany, and the U.K. He has published over 220 papers in IEEE TRANSACTIONS and over 60 papers in Automatica. His research interests include dynamical systems, signal processing, bioinformatics, control theory and applications.

Prof. Wang is a holder of the Alexander von Humboldt Research Fellowship of Germany, the JSPS Research Fellowship of Japan, William Mong Visiting Research Fellowship of Hong Kong. He serves (or has served) as the Editor-in-Chief for Neurocomputing, the Deputy Editor-in-Chief for the International Journal of Systems Science, and an Associate Editor for 12 international journals, including the IEEE TRANSACTIONS ON AUTOMATIC Control, IEEE TRansactions on CONTROL System TeChNology, IEEE TRANSACTIONS ON NEURAL NETWORKS, IEEE TRANSACTIONS ON Signal Processing, and IEEE TRANSACTIONS ON SYSTEMS, MAN, AND Cybernetics: Systems. He is a fellow of the Royal Statistical Society and a member of program committee for many international conferences.

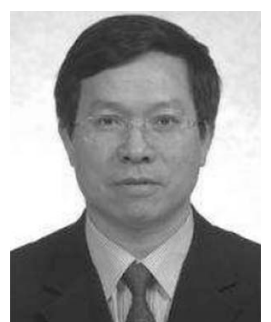

Guo-Ping Liu (Fellow, IEEE) received the B.Eng. and M.Eng. degrees in automation from the Central South University, Changsha, China, in 1982 and 1985, respectively, and the Ph.D. degree in control engineering from the University of Manchester, Manchester, U.K., in 1992.

$\mathrm{He}$ is a Professor with the University of South Wales, Pontypridd, U.K. and is a Visiting Professor with Wuhan University, Wuhan, China. He has authored/coauthored over 200 journal papers and 10 books on control systems. His current research interests include networked multiagent control systems, nonlinear system identification and control, advanced control of industrial systems, and multiobjective optimization and control.

Prof. Liu is an Editor-in-Chief of the International Journal of Automation and Computing and an IET Fellow.

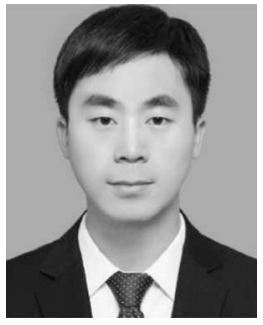

Hongxu Zhang received the B.Sc. degree in information and computation science and the M.Sc. degree in mathematics from the Harbin University of Science and Technology, Harbin, China, in 2014 and 2017, respectively, where he is currently pursuing the Ph.D. degree in measuring and testing technologies and instruments with the School of Measurement and Communication.

His research interests include optimal state estimation and sliding mode control for complex dynamical systems.

Mr. Zhang is an active reviewer for many international journals

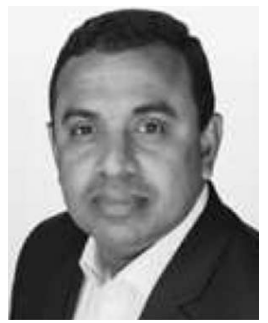

Rukshan Navaratne received the Ph.D. degree in aerospace engineering from Cranfield University, Cranfield, U.K.

Before joining academia, he has spent 18 years of his career working as a Design Engineer with Toyota Motor Corporation, Ohira, Japan, and a Senior Manager with MTU Aero Engines, Munich, Germany. He is currently working as a Senior Lecturer with the School of Engineering, University of South Wales, Pontypridd, U.K. He uses variety of numerical and experimental tools and techniques to develop propulsion technologies from initial conception through increasing levels of technology readiness with a constant view toward commercialization and real world use. His current research focuses on the development of novel electric propulsion systems, electrical machine modeling, and instrumentation.

Dr. Navaratne is a Charted Engineer and a member of IMechE (U.K.) 